\title{
Inertialess multilayer film flow with surfactant: stability and travelling waves
}

\author{
J. Thompson and M. G. Blyth* \\ School of Mathematics, University of East Anglia, \\ Norwich Research Park, Norwich, NR4 7TJ, UK.
}

(Dated: September 13, 2016)

\begin{abstract}
Multilayer film flow down an inclined plane in the presence of an insoluble surfactant is investigated with particular emphasis on determining flow stability and investigating the possibility of travelling wave solutions. The investigation is conducted for two or three layers under conditions of Stokes flow and, separately, on the basis of a long-wave assumption. A normal mode linear stability analysis for Stokes flow shows that adding surfactant to one of the film surfaces can destabilise an otherwise stable flow configuration. For the long-wave system, periodic travelling-wave branches are detected and traced, revealing solutions with pulse-like solitary waves on each film surface travelling in phase with each other, travelling waves with capillary ridge structures, and solutions with two of the film surfaces almost in contact. Time-periodic travelling wave solutions are also found. The stability of the travelling waves is determined by solving initial value problems and by computing eigenvalue spectra. Boundary element simulations for Stokes flow confirm the existence of travelling waves outside of the longwave regime.
\end{abstract}

\section{INTRODUCTION}

In this paper we examine the flow of superposed layers of viscous fluid down an inclined plane when the interfaces between the layers, and the free surface, are covered with an insoluble surfactant. Multilayer film flows of this type are of considerable interest theoretically and occur in a range of industrial applications, notably film coating technologies. In optical coating, for example, a target surface such as a spectacle lens is coated with one or more liquid layers whose thicknesses are precision-engineered to maximise anti-reflection capability [e.g. 1]. Hydrophilic film coating of medical devices such as catheters is used to enhance lubricity and provide bio-active functionality [e.g. 2]. In many applications, uniform layer thickness is of the utmost importance and waves and other disturbances must be eliminated. One approach to minimise such disruptions is to introduce an insoluble surfactant, working on the intuitive basis that surfactant lowers the local interfacial tension between fluids and hence acts to reduce any capillary instability. However, surfactant may promote instability or even destabilise an otherwise stable system.

The dynamics of a clean (i.e. a surfactant-free) layer flowing down an inclined plane was discussed by Benjamin [3] and Yih [4]. In the simplest configuration the free surface of the film is flat and the velocity in the fluid adopts the classical unidirectional, semi-parabolic Nusselt profile. Under perturbation, in the absence of inertia there is a single normal mode, which is found to be negative corresponding to a stable flow. Long-wave instability occurs when the Reynolds number exceeds a threshold value. This was confirmed experimentally by Liu et al. [5]. Following Whitaker [6], who worked with a set of approximate boundary conditions, Whitaker [7]

*m.blyth@uea.ac.uk and Lin [8] extended Yih's long-wave analysis to allow for the effect of a surfactant. With surfactant present, the critical Reynolds number is larger and so the surfactant has a stabilising effect. This result was obtained for a vertically falling film by Anshus and Acrivos [9], who also showed that the wavelength of the most dangerous mode is significantly increased by surfactant. Ji and Setterwall [10] discussed the effect of soluble surfactant and demonstrated the existence of an unstable Marangoni mode for a vertical film. Pozrikidis [11] relaxed the long-wave assumption adopted by previous workers and showed that for a single film in the inertialess, zero Reynolds number limit, there are two normal modes, the first of which corresponds to that found by Yih [4] and the second of which is a Marangoni mode contributed by the surfactant. Both of these modes are stable at zero Reynolds number. Blyth and Pozrikidis [12] solved the Orr-Sommerfeld problem for a contaminated film numerically and showed that either the Yih mode or the Marangoni mode can be unstable in the presence of inertia, and one or the other dominates depending on the size of the Reynolds number.

Remarkably, instability can be found for two-layer flow even at zero Reynolds number. In the basic state, the interface between the fluids and the free surface are flat and the velocity field is unidirectional. Working in the longwave limit in which interfacial tension and surface tension are both negligible, Kao [13] showed that for clean twolayer flow the basic state is always unstable when there is no inertia and when the upper layer is more viscous than the lower layer. This result was extended to the case of arbitrary wavenumber in the Stokes flow limit, and in the absence of interfacial and surface tension, by Loewenherz and Lawrence [14]. Further analysis by Chen [15] allowed for surface tension and inertia and concluded that the clean two-layer flow is always unstable at any Reynolds number if the less viscous fluid is next to the wall. Jiang and Lin [16] showed that the unstable two-layered flow on 
a vertically inclined plate can be stabilised by oscillating the plate in its own plane. Fully nonlinear computations for a clean two-layer system moving under conditions of Stokes flow were performed by Pozrikidis [17] using the boundary integral method. Jiang et al. [18] and Gao and $\mathrm{Lu}$ [19] have discussed the physical origin of the inertialess two-layer instability. A set of model equations for clean, two-layer flow, which is derived on the basis of the lubrication approximation, and which incorporates the effect of inertia, has been used by Boutounet et al. [20] to demonstrate the occurrence of roll waves for an unstable configuration.

Gao and $\mathrm{Lu}[21]$ studied the linear stability of twolayer flow in the presence of surfactant. They conducted a normal mode linear stability analysis in the limit of zero Reynolds number for arbitrary wavelength disturbances. With surfactant present there are four possible normal modes. Assuming layers of equal thickness they found that at most one of these modes is unstable. They showed that for a stable, clean configuration with the more viscous fluid next to the wall, the flow remains stable when surfactant is added to the free surface but is destabilised when surfactant is added to the interface. For an unstable clean configuration with the less viscous fluid adjacent to the wall, and for a moderate size viscosity ratio, introducing surfactant to the free surface reduces the cut-off wavenumber for instability and decreases the maximum growth rate. However, introducing surfactant to the interface raises or lowers the maximum growth rate depending on the size of the interfacial Marangoni number. Recently Samanta [22] has extended the analysis to include the effect of inertia.

Clean three-layer flow has been investigated by a number of workers. Following work by Akhtaruzzaman [23] on three-layer flow, Wang and Seaborg [24] identified long-wave instability due to viscosity stratification but only when inertia is included. Later, also working on the basis of long wavelength perturbations, Weinstein and Kurz [25] documented the various effects of viscosity stratification, density stratification, and different layer thicknesses, and concluded that three-layer flow can be unstable even at zero Reynolds number, and specifically when the middle layer is thin and less viscous than either of the adjacent layers. Kliakhandler and Sivashinsky [26] also found instability in a clean three-layer system in the long-wave limit and likened it to the kinetic alpha effect in magnetohydrodynamics. Weinstein and Chen [27] examined disturbances under conditions of Stokes flow, including the effect of surface tension but neglecting any interfacial tensions. They noted in particular that the instability identified by Weinstein and Kurz [25] is still present when the longwave approximation is relaxed. They also identified a new instability which is not present in the longwave regime, which occurs when the middle layer is thin and highly viscous. Furthermore, they showed that the largest growth rates found for the three-layer system are orders of magnitude larger than those found for the two-layer system by Loewenherz and Lawrence [14]. The physical mechanisms behind the three-layer instability was investigated by Jiang et al. [28]. Weakly nonlinear models for three-layer flow have been proposed by Kliakhandler and Sivashinsky ([29], [30]). However, the derivation of these weakly nonlinear models has been criticised ([31]).

In the present work, we re-examine the case of twolayer flow with surfactant and study, for the first time to our knowledge, three-layer flow in the presence of surfactant. The paper is organised as follows. In sections II and III we describe the problem formulation and discuss the linear stability problem for three-layers. In section IV we derive a model system of equations describing two or three layer flow working on the basis of the lubrication approximation. Nonlinear solutions of these equations are described including travelling-wave solutions, and the stability of these solutions is discussed. In section $\mathrm{V}$ we present some nonlinear calculations performed under conditions of Stokes flow using the boundary-element method. Finally, in section VII we summarise our findings and discuss future directions.

\section{PROBLEM FORMULATION}

We consider the gravity-driven flow of superposed layers of viscous fluid down a plane inclined at an angle $\theta$ to the horizontal, as is sketched in figure 1. The sketch shows three-layered flow but two-layer flow is also considered. The $x$ and $y$ axes are respectively parallel to and normal to the plane. The films are labelled by the index $j=1,2,3$ with the lowermost film corresponding to $j=1$ being adjacent to the wall. The fluid in film $j$ has density $\rho_{j}$ and viscosity $\mu_{j}$. The interfaces between the films and the free surface are covered with insoluble surfactant which acts to modify the interfacial tensions and the surface tension according to the local concentration through an assumed equation of state to be described below. Henceforth, interface 1 refers to the interface between film 1 and 2, and interface 2 to that between film 2 and 3.

The flow in the films is assumed to satisfy the linear equation of Stokes flow, so that

$$
\mathbf{0}=-\nabla p_{j}+\rho_{j} \mathbf{g}+\mu_{j} \nabla^{2} \mathbf{u}_{j}, \quad \nabla \cdot \mathbf{u}_{j}=0,
$$

for $j=1,2,3$, where $\mathbf{g}=g(\sin \theta,-\cos \theta)$ and $g$ is the gravitational acceleration, and $\mathbf{u}_{j}=\left(u_{j}, v_{j}\right)$ is the fluid velocity in the $j$ th film. At the interfaces and at the free surface, the fluid stress experiences a jump discontinuity due to the interfacial/surface tension, which is given by

$$
\left(\boldsymbol{\sigma}^{(j)}-\boldsymbol{\sigma}^{(j+1)}\right) \cdot \mathbf{n}_{j}=\gamma_{j} \kappa_{j} \mathbf{n}_{j}-\frac{\partial \gamma_{j}}{\partial l} \mathbf{t}_{j}
$$

where $\boldsymbol{\sigma}^{(j)}$ is the Newtonian stress tensor in film $j=$ $1,2,3$, with $\boldsymbol{\sigma}^{(4)}=-p_{a}^{*} \mathbf{I}$, where $p_{a}^{*}$ is atmospheric pressure. The interfacial tensions at interface 1 and 2 are $\gamma_{1}$, $\gamma_{2}$ respectively, and the surface tension is $\gamma_{3}$. The unit normal vectors $n_{j}$ point downwards into fluid $j-1$, and 


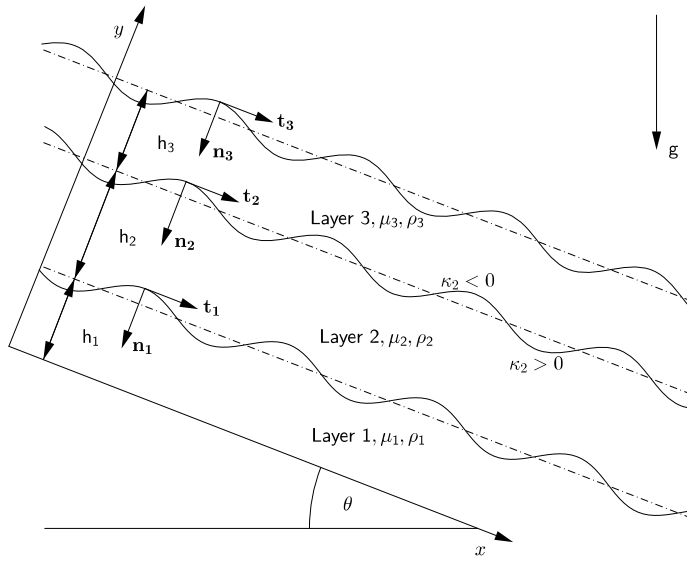

FIG. 1. Illustration of a three layer flow down an inclined plane. Interface $j$ lies between layers $j$ and $j+1$ for $j=$ 1,2 . The sign of the curvature is shown on interface 2 for illustration. Both two and three-layer flow is considered in this paper.

the unit tangent vectors point in the direction of increasing arc length $l$. The curvatures at the interfaces and at and free surface, $\kappa_{j}=-\mathbf{n}_{j} \cdot \mathrm{d} \mathbf{t}_{j} / \mathrm{d} l$, are defined to be positive when the respective surface is concave upwards, as is illustrated in figure 1. The kinematic condition at each surface requires that

$$
\frac{\mathrm{D}}{\mathrm{D} t}\left(y-y_{j}\right)=0
$$

where $\mathrm{D} / \mathrm{D} t$ is the material derivative, and $y_{j}$ are the locations of interface 1 , interface 2 , and the free surface respectively.

Assuming that the surfactant concentrations $\Gamma_{j}$ at the two interfaces $(j=1,2)$ and at the free surface $(j=3)$ are sufficiently dilute, it is reasonable to adopt the linear equation of state (e.g. [32], [33])

$$
\gamma_{j}=\gamma_{j c}\left(1-\beta_{j} \frac{\Gamma_{j}}{\Gamma_{j 0}}\right),
$$

for $j=1,2,3$, where $\gamma_{j c}$ is the prevailing tension in the absence of surfactant for a clean interface/free surface, and $\Gamma_{j 0}$ are reference levels corresponding to the tensions $\gamma_{j 0}=\gamma_{j c}\left(1-\beta_{j}\right)$. The dimensionless parameters $\beta_{j}$ are related to the interfacial/free surface Marangoni numbers by

$$
M a_{j}=\frac{\beta_{j}}{1-\beta_{j}} .
$$

In the remainder of the paper, the effects of surfactant will be quantified with reference to the Marangoni numbers $M a_{j}$.

The surfactant is convected and diffuses over the interfaces and over the free surface according to the transport equation (e.g. [34])

$$
\frac{\mathrm{d} \Gamma_{j}}{\mathrm{~d} t}+\frac{\partial\left(u_{j}^{t} \Gamma_{j}\right)}{\partial l}-\tilde{w}_{j} \frac{\partial \Gamma_{j}}{\partial l}+\kappa_{j} u_{j}^{n} \Gamma_{j}=D_{j} \frac{\partial^{2} \Gamma_{j}}{\partial l^{2}},
$$

where $u_{j}^{t}=\mathbf{u}_{j} \cdot \mathbf{t}_{j}$ and $u_{j}^{n}=\mathbf{u}_{j} \cdot \mathbf{n}_{j}$ are, respectively, the tangential and normal components of the interfacial and free surface velocities $\mathbf{u}_{j}$, and the $D_{j}$ are surfactant diffusivities. The derivative $\mathrm{d} / \mathrm{d} t$ on the left-hand side of (6) is the rate of change following the motion of interfacial marker points moving with the normal component of the fluid velocity at the interface and with a tangential velocity $\tilde{w}_{j}$ which may be chosen arbitrarily (for example, if we take $\tilde{w}_{j}=u_{j}^{t}$, the marker points move with the local fluid velocity).

It will be convenient to nondimensionalise variables using the unperturbed thickness of the bottom film, $h_{1}$, as the lengthscale, and $\hat{U}$ as the velocity scale, $\mu_{1} \hat{U} / h_{1}$ as the pressure scale and $h_{1} / \hat{U}$ as the time scale, where $\hat{U}=\rho_{1} g h_{1}^{2} \sin \theta / \mu_{1}$ is twice the classical Nusselt surface speed for a single, flat film flowing down an inclined plane. The surface and interfacial tensions and the respective surfactant concentrations are made dimensionless using the values $\gamma_{j 0}$ and $\Gamma_{j 0}$. Accordingly we introduce the dimensionless capillary numbers and Péclet numbers,

$$
C a_{j}=\mu_{j} \hat{U} / \gamma_{j 0}, \quad P e_{j}=\frac{h_{1} \hat{U}}{D_{j}}
$$

for $j=1,2,3$.

In the simplest configuration, the interfaces and the free surface are flat so that all of the films are of uniform, but generally different, thickness, the flow in each film is unidirectional along the $x$-axis, and the velocity field is continuous at each interface. The nondimensionalised unidirectional velocity field in layer $j=1,2,3$ is given by $\mathbf{u}_{j}=\bar{u}_{j}(y) \mathbf{i}$, where

$$
\begin{gathered}
\bar{u}_{1}=-\frac{1}{2} y^{2}+a_{1} y, \quad \bar{u}_{2}=-\frac{R_{2}}{2 m_{2}} y^{2}+a_{2} y+b_{2}, \\
\bar{u}_{3}=-\frac{R_{3}}{2 m_{3}} y^{2}+a_{3} y+b_{3},
\end{gathered}
$$

where

$$
\begin{gathered}
a_{1}=1+r_{2} R_{2}+r_{3} R_{3}, \quad m_{2} a_{2}=\left(1+r_{2}\right) R_{2}+r_{3} R_{3}, \\
m_{3} a_{3}=\left(1+r_{2}+r_{3}\right) R_{3},
\end{gathered}
$$

and

$$
\begin{aligned}
b_{2}=r_{3} & \left(1-1 / m_{2}\right) R_{3}+ \\
& {\left[r_{2}-\left(1+2 r_{2}\right) /\left(2 m_{2}\right)\right] R_{2}+1 / 2, }
\end{aligned}
$$

and

$$
\begin{aligned}
b_{3}=r_{2}[1+ & \left.r_{2} /\left(2 m_{2}\right)\right] R_{2}+1 / 2 \\
& +\left[r_{3}\left(1+r_{2} / m_{2}\right)\right. \\
& \left.-\left(1+r_{2}\right)\left(1+r_{2}+2 r_{3}\right) /\left(2 m_{3}\right)\right] R_{3} .
\end{aligned}
$$

Here

$$
\begin{gathered}
\left(m_{2}, m_{3}\right)=\frac{1}{\mu_{1}}\left(\mu_{2}, \mu_{3}\right), \quad\left(r_{2}, r_{3}\right)=\frac{1}{h_{1}}\left(h_{2}, h_{3}\right), \\
\left(R_{2}, R_{3}\right)=\frac{1}{\rho_{1}}\left(\rho_{2}, \rho_{3}\right)
\end{gathered}
$$


are the viscosity ratios, the layer thickness ratios, and the density ratios respectively. The dimensionless base flow pressure field is given by

$\bar{p}_{1}=-y \cot \theta+\left(1+\left(r_{2}-1\right) R_{2}+\left(r_{3}-r_{2}\right) R_{3}\right) \cot \theta+p_{a}$,

$\bar{p}_{2}=-y R_{2} \cot \theta+\left(r_{2} R_{2}+\left(r_{3}-r_{2}\right) R_{3}\right) \cot \theta+p_{a}$,

$\bar{p}_{3}=-y R_{3} \cot \theta+r_{3} R_{3} \cot \theta+p_{a}$,

where $p_{a}$ is the dimensionless atmospheric pressure, whose value is immaterial for the dynamics.

\section{LINEAR STABILITY: STOKES FLOW}

To assess the linear stability of the simple, unidirectional flow configuration, we carry out a normal mode analysis. We perturb the interface locations, $y_{1}(x, t)$ and $y_{2}(x, t)$, and the free surface location $y_{3}(x, t)$, so that

$$
y_{1}=1+\epsilon A_{1} \mathrm{e}^{\mathrm{i} k(x-c t)}, y_{l}=y_{l 0}+\epsilon A_{l} \mathrm{e}^{\mathrm{i} k(x-c t)},
$$

for $l=2,3$, where $y_{l 0}=1+\sum_{j=2}^{l} r_{j}$. Here and below the addition of the complex conjugate to the perturbation terms is understood. The generally complex amplitudes $A_{1}, A_{2}$ and $A_{3}$ and the complex wavespeed $c$ are to be determined for a given value of the perturbation wave number $k$. We perturb the flow quantities by writing

$$
\left(\psi_{j}, p\right)=\left(\bar{\psi}_{j}, \bar{p}\right)+\epsilon\left(\phi_{j}(y), q_{j}(y)\right) \mathrm{e}^{i k(x-c t)},
$$

where $\psi_{j}$ is the streamfunction defined so that $u_{j}=$ $\partial \psi_{j} / \partial y$ and $v_{j}=-\partial \psi_{j} / \partial x$. The form of the base flow streamfunction $\bar{\psi}_{j}$ can be readily found using the basic velocity field given in (8). Similarly, we expand the surface tensions and surfactant concentrations by writing

$$
\left(\gamma_{j}, \Gamma\right)=(1,1)+\epsilon\left(g_{j}, G_{j}\right) \mathrm{e}^{i k(x-c t)} .
$$

Substituting these forms in the Stokes equations and boundary conditions we ultimately derive a sixth order polynomial for the complex growth rate $c$. The details of this calculation are given in the Appendix. The six modes corresponding to the six values of $c$ comprise three surface modes representing deformations to either of the two interfaces or the free surface, and three Marangoni modes associated with perturbations to the surfactant distribution on each of the surfaces.

For a fixed wavenumber $k$, the normal modes depend on the 13 parameters comprising the film thickness ratios $r_{2}$ and $r_{3}$, the viscosity ratios $m_{2}$ and $m_{3}$, the density ratios $R_{2}$ and $R_{3}$, the Marangoni numbers $M a_{1}, M a_{2}$ and $M a_{3}$, the capillary numbers $C a_{1}, C a_{2}$ and $C a_{3}$ and the inclination angle $\theta$. To reduce the dimension of the parameter space, we henceforth consider fluids of equal density so that $R_{2}=R_{3}=1$. We focus attention in particular on the effect of the surfactant at the fluid interfaces and on the free surface, as this is expected to affect the flow stability. Previous studies have also shown that the viscosity contrast between the layers can play an important role in deciding stability. We have validated our calculations by successfully comparing our results with those of Gao and $\mathrm{Lu}$ [21] in the case of two layers with surfactant, and with those of Weinstein and Chen [27] for three clean layers.

\section{A. Two-layer flow}

The case of two-layer flow was studied in detail by Gao and $\mathrm{Lu}[21]$ for layers of equal thickness. Assuming equal densities, a clean two-layer flow is stable when the more viscous layer is next to the wall, and is unstable when the less viscous film is next to the wall so that $m_{2}>1$. Gao and $\mathrm{Lu}[21]$ demonstrated that a stable, clean two-layer film with $m_{2}<1$ is destabilised by adding surfactant to the interface between the films. However, the flow remains stable when surfactant is added to the free surface, and they found no evidence that surfactant can stabilise a unstable clean configuration. Our findings confirm these observations but, in addition, we find that adding surfactant to the free surface can destabilise a clean two-layer flow with $m_{2}<1$ if the upper layer is thinner than the lower layer so that $r_{2}<1$. Figure 2 shows neutral curves dividing regions of stability and instability for a sample case with a thinner upper layer. It is clear from figure 2 (a) that even a small amount of surfactant on the interface destabilises the flow for any viscosity ratio. The topology of the neutral curves is quite different, however, when surfactant is added to the free surface. Figure $2(b)$ reveals a closed island of unstable modes in the region $m_{2}<1$, confirming the above observation that a clean, stably stratified flow can be destabilised by adding surfactant onto the free surface. In this case the upper layer is thinner than the lower layer and we note that the island of unstable modes vanishes if the upper layer is taken to be the thicker one. These figures also reveal that the character of the instability for a more viscous lower layer $\left(m_{2}<1\right)$ is quite different when surfactant is on the interface or on the free surface. In the former case, long-wave instability occurs, and in the latter case, the instability occurs over a band of finite, non-zero wavenumbers. As a further point of interest, we note from figure $2(a)$ that when $M a_{1} \neq 0$ and for sufficiently large $m_{2}$, there are two disjoint bands of unstable wavenumbers corresponding to two different and simultaneously unstable linear modes. The growth rates for these modes are shown in figure $2(c)$ for $m_{2}=2.5$. This is a facet of differential layer thicknesses; when $r_{2}=1$ Gao and $\mathrm{Lu}$ [21] noted that at most one of the four possible modes (in the presence of surfactant) can be unstable for a fixed parameter set. 
(a)

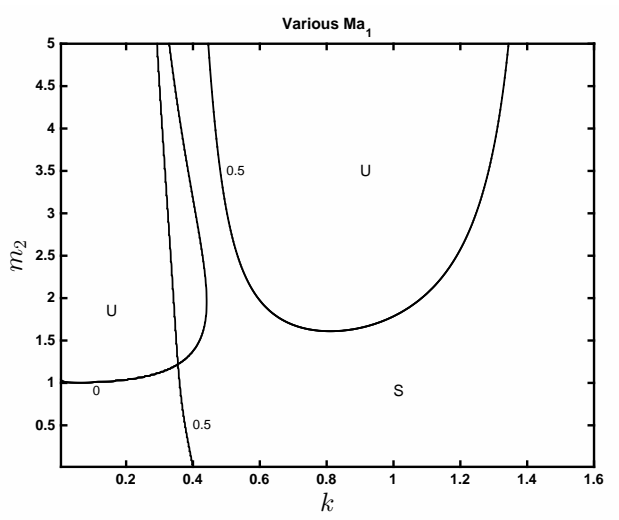

(b)

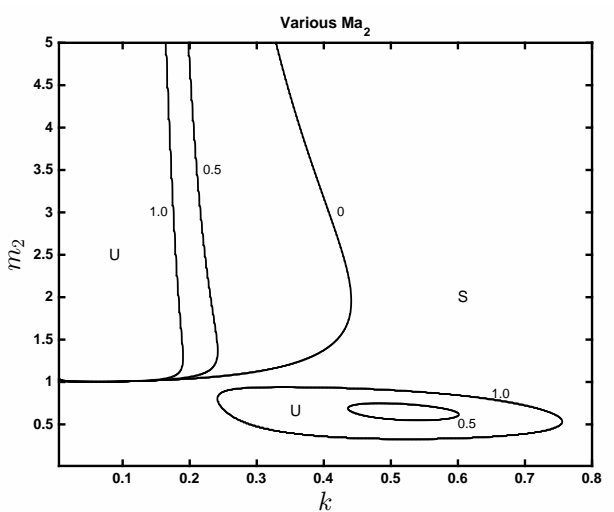

(c)

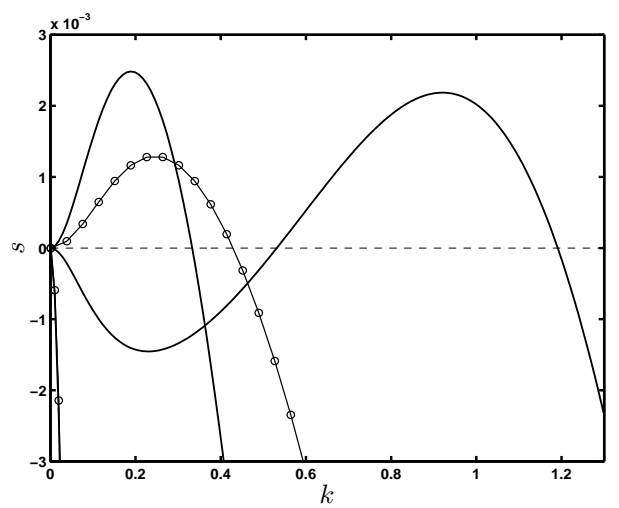

FIG. 2. Two-layer flow: $(a-b)$ Neutral curves for the case $\theta=0.2, C a_{1}=C a_{2}=1, r_{2}=0.5, R_{2}=1$ with $(a) M a_{2}=0$, various $M a_{1}$ and (b) $M a_{1}=0$, various $M a_{2}$. Regions are marked stable (S) and unstable (U). (c) Growth rates for (a) when $m_{2}=2.5, M a_{2}=0.5$. The two growth rates for clean layers $\left(M a_{1}=M a_{2}=0\right)$ are shown with lines marked with circles. One of the growth rates is identically zero (since $\left.M a_{1}=0\right)$ and is not shown; the stable mode is almost coincident with one of the two clean modes.

\section{B. Three-layer flow}

A clean three-layer system may also be unstable due to viscosity stratification. Excluding cases where one or more layers have equal viscosity, there are six possible distinct configurations. Writing using the layer number in order of increasing viscosity, these are given by (i) $\{1,2,3\},($ ii $)\{1,3,2\}$, (iii) $\{2,1,3\},($ iv $)\{2,3,1\},(v)$ $\{3,1,2\}$, (vi) $\{3,2,1\}$. On the basis that having a less viscous layer underneath a more viscous layer is likely to give an unstable system, we expect only configuration (vi) to be stable and our computations for a clean system confirm this to be the case. In particular, in configuration $(i)$ there are two unstable normal modes across the wave number range, and there is one unstable mode for configurations $(i i),(i i),(i v)$ and $(v)$.

Assuming zero interfacial tensions, the growth rates for a three-layer system are substantially higher than those for a two-layer system ([27]). Figure 3(a) shows our recomputed results at the parameter values used by [27] in their figure 3, and illustrates the effect of adding surfactant. Evidently the surfactant introduces a new mode, which is always stable. Although the surfactant destabilises small wavelength perturbations, its influence over larger wavelength disturbances is essentially stabilising. Note that we do not find it to be generally true that the three-layer growth rates are much larger than two-layer growth rates when the interfacial capillary numbers are finite, as will be discussed in the following paragraphs. For vanishing interfacial tensions, Weinstein and Chen [27] also identified a new instability with moderate wavelength for three-layer flow characterised by near-complexconjugate modes (see their figure 2, for example). An example of this is illustrated in figure 3(b), where the effect of adding surfactant to the free surface is also calculated. As for the case in figure 3(a), the surfactant reduces the growth rate of the unstable modes.

Adding surfactant to either of the interfaces or to the free surface can destabilise a clean system, depending on the relative thicknesses of the layers. We found no evidence that surfactant can stabilise an unstable clean configuration. In figure 4 we show neutral curves for a system with the upper two layers of equal thickness and half the thickness of the lowermost layer. This system is stable when clean $\left(M a_{1}=M a_{2}=M a_{3}=0\right)$ provided the viscosity ratio $m_{3}<0.5$. The three panels show regions of stability and instability when surfactant is added to either of the two interfaces or to the free surface. Evidently in each case it is possible to destabilise the stable, clean system. As for the two-layer flow case shown in figure 2, the structure of the neutral curves is different when surfactant is on the free surface (figure 4c). In this case there is a disconnected island of unstable modes in the region $m_{3}<0.5$. The flow is stable for some values of $m_{3}<0.5$ even with surfactant on the interface. As for the two-layer case, the unstable island is present as a result of different layer thicknesses. If the layer thickness are all taken to be the same, this island vanishes and the flow is stable for $m_{3}<0.5$.

Stability regions for a sample parameter set are shown in figure 5 for different viscosity ratios and different layer thicknesses. The parameters are such that for the right 
(a)

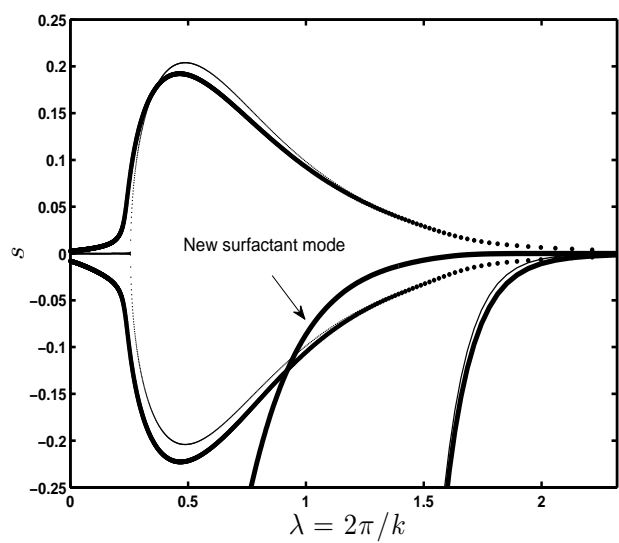

(b)

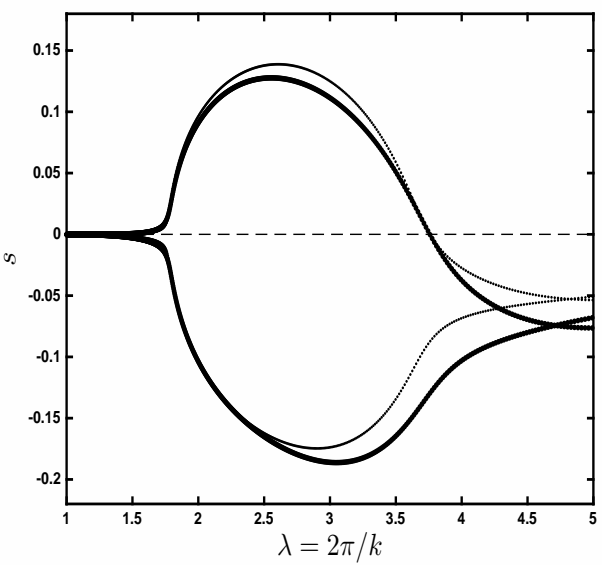

FIG. 3. Growth rates for three-layer flow with $\theta=\pi / 2, C a_{1}=$ $C a_{2}=\infty, m_{3}=1, R_{2}=R_{3}=1$, and $M a_{1}=M a_{2}=0$. (a), $C a_{3}=0.001, r_{2}=0.05 / 0.475, r_{3}=1, m_{2}=0.2, M a_{3}=$ 0.001. (b) $C a_{3}=1, r_{2}=0.5, r_{3}=1, m_{2}=10$. In (a) the thinner lines show [27]'s results for $M a_{3}=0$ ). In (b) the thin lines are for $M a_{3}=0$ and thick lines for $M a_{3}=1$.

choice of $m_{2}, m_{3}$ and $r_{2}, r_{3}$ we recover the case studied in figure 4 . Referring to figure $5(a)$, for the clean system the flow is stable when $m_{3}<m_{2}$ and $m_{2}<1$ and $m_{3}<1$ so that the stability region on a set of $m_{2}-m_{3}$ axes is triangular. With either $M a_{1}$ or $M a_{2}$ non-zero, the flow is unstable for all $m_{2}, m_{3}$; however a portion of the stable triangular region remains as $M a_{3}$ is increased from zero. For figure $5(b)$ the clean system is stable for all $r_{2}, r_{3}$. With $M a_{3}$ non-zero an unstable region appears as shown, whose thickness increases as $M a_{3}$ is increased (the flow is everywhere unstable in the $r_{2}-r_{3}$ plane when either $M a_{1} \neq 0$ or $M a_{2} \neq 0$ ).

In all of our investigations we found at most three unstable normal modes over the wave number range for a given parameter set. Figure 6 shows an example where three of the possible six normal modes are unstable. Note that since $M a_{1}=M a_{3}=0$ two of the six growth rates are identically zero, $s=0$. The clean system is an un- (a)

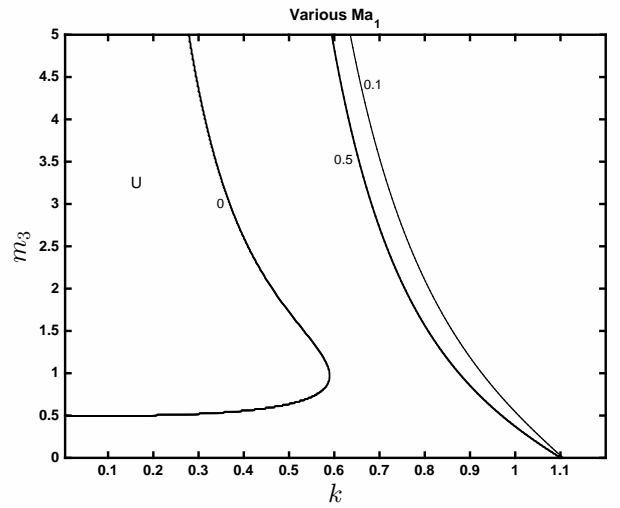

(b)

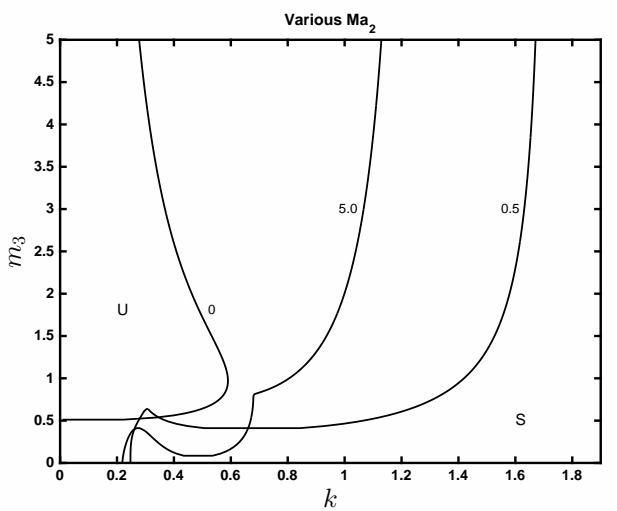

(c)

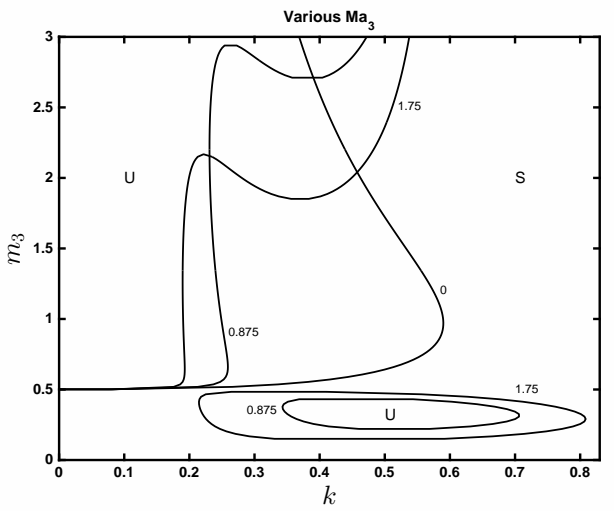

FIG. 4. Neutral curves for three-layer flow with $\theta=0.2$, $C a_{1}=C a_{2}=C a_{3}=1, r_{2}=r_{3}=0.5, m_{2}=0.5, R_{2}=$ $R_{3}=1$ with (a) $M a_{2}=M a_{3}=0$, various $M_{1}$, and (b) $M a_{1}=M a_{3}=0$, various $M_{2}$, and (c) $M a_{1}=M a_{2}=0$, various $M_{3}$. Regions are marked stable (S) and unstable (U).

stable configuration of type $(i)$ and therefore, as noted above, has two unstable normal modes. These are shown as lines marked with circles in figure 6 . The effect of adding surfactant onto the second interface, and increasing $M a_{2}$ from zero to 0.001 (see figure $6 a$ ), is to lower the maximum growth rate of one of the two unstable clean modes and to increase the maximum growth rate of the other; also the cut-off wavenumber of one of the clean 
(a)
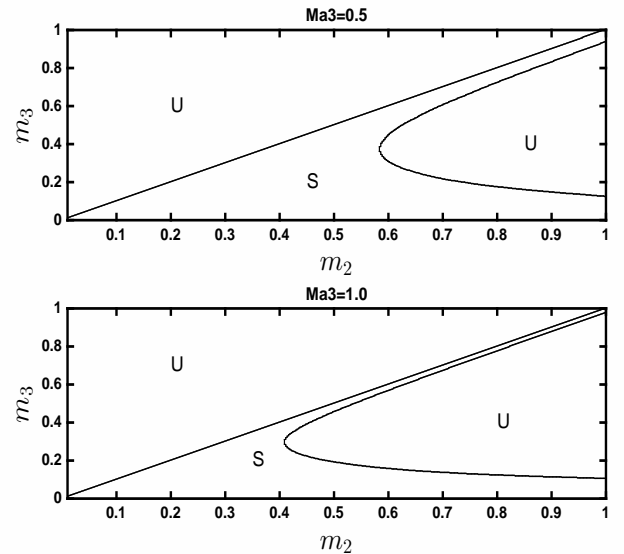

(b)
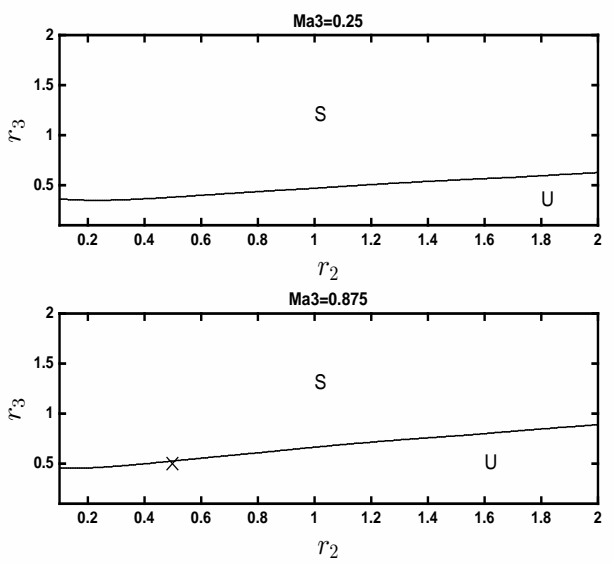

FIG. 5. Stability regions (U for unstable; $\mathrm{S}$ for stable) for three-layer flow for the case $\theta=0.2, C a_{1}=C a_{2}=C a_{3}=1$, $R_{2}=R_{3}=1, M a_{1}=M a_{2}=0$ and various $M a_{3}:(a) m_{2}$ versus $m_{3}$ for $r_{2}=r_{3}=0.5$, and (b) $r_{2}$ versus $r_{3}$ for $m_{2}=0.5$, $m_{3}=0.3$. The cross in the lower panel indicates the unstable case considered in figure 4 .

modes is lowered while the other is raised. At the same time, a new surfactant mode comes into play and opens up a band of unstable wavenumbers excluding zero. This is more clearly seen in 6(b) for the larger Marangoni number $M a_{2}=0.5$. In this case the surfactant mode dominates, contributing the largest growth rate. Note that the cut-off wavenumber for the second unstable clean mode is now lower than its clean value, which indicates a nonmonotonic dependence on the Marangoni number. Similarly, the growth rate of the second clean mode is lower, again indicating non-monotonic behaviour.

\section{LONG-WAVE APPROXIMATION}

In this section we analyse two-layer and three-layer flow on the assumption that variations in the streamwise direction are much slower than across the films. We (a)

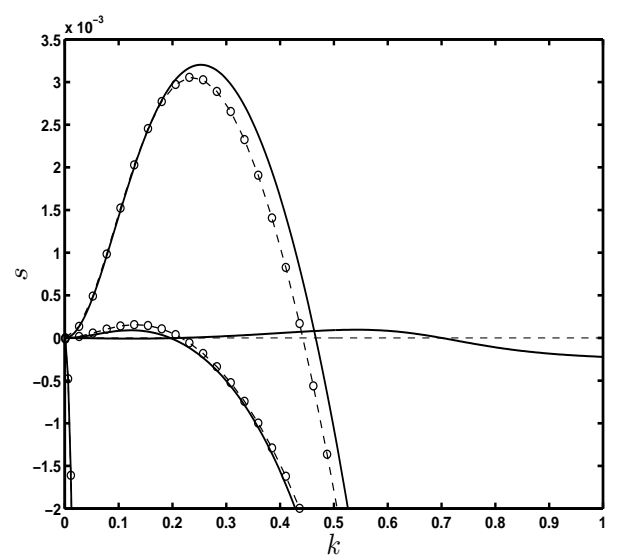

(b)

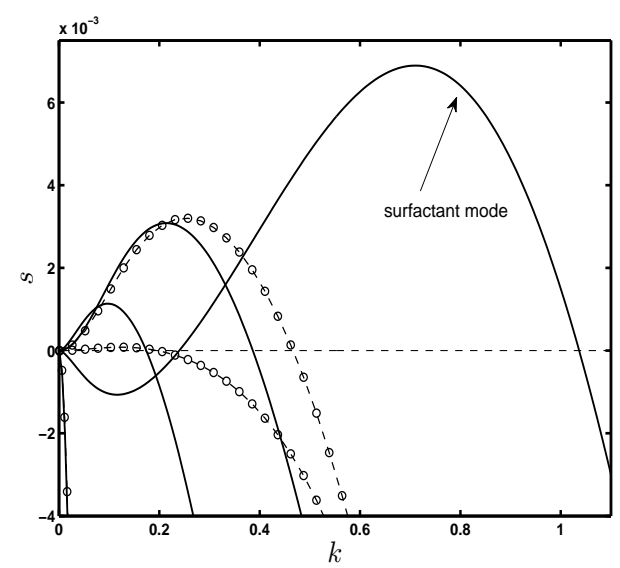

FIG. 6. Three-layer Stokes flow growth rates $s=\operatorname{Im}(k c)$ for $\theta=0.2, C a_{1}=C a_{2}=C a_{3}=1, r_{2}=r_{3}=0.5, m_{2}=1.5$, $m_{3}=2.0, R_{2}=R_{3}=1, M a_{1}=M a_{3}=0$ and $(a) M a_{2}=$ 0.001 and (b) $M a_{2}=0.5$. In both panels the rates found for a clean system with the same parameter values are shown with broken lines marked with circles.

discuss the linear stability under this assumption, and compute nonlinear travelling wave solutions and examine their stability.

We describe the interfaces and the free surface by the explicit forms $y_{j}(x, t)=F_{j}(x, t)$. The surfactant transport equation (6) then adopts the equivalent form ([35])

$$
\frac{\partial\left(s_{j} \Gamma_{j}\right)}{\partial t}+\frac{\partial\left(s_{j} \Gamma_{j} u_{j}^{s}\right)}{\partial x}=\frac{1}{P e_{j}} \frac{\partial}{\partial x}\left(\frac{1}{s_{j}} \frac{\partial \Gamma_{j}}{\partial x}\right),
$$

where $s_{j}=\left(1+F_{j x}^{2}\right)^{1 / 2}$ and $u_{j}^{s}=u_{j}\left(x, F_{j}\right)$, and on noting that we have made the choice $\tilde{w}_{j}=u_{j}^{t}$ for the arbitrary tangential velocity.

We describe the derivation of the long-wave system of equations for three layer flow - the two-layer case is an obvious reduction of this. We nondimensionalize using the same scales used in section II. Introducing the small long-wave parameter $\delta \ll 1$, we define the slow spatial 
and time variables $\xi=\delta x$ and $\tau=\delta t$ and expand variables by writing

$$
\begin{aligned}
& F_{j}=f_{j}+\cdots, \quad u_{j}=U_{j}+\cdots, \quad v_{j}=\delta V_{j}+\cdots, \\
& p_{j}=R_{j}(x-y \cot \theta)+\delta^{-1} P_{j}+\cdots,
\end{aligned}
$$

where $R_{1}=1$, and $R_{2}, R_{3}$ were defined in (12). Note that $f_{j}, U_{j}, W_{j}$ and $P_{j}$ are all $O(1)$ quantities; moreover we assume that $\Gamma_{j}$ is $O(1)$. We further assume that $\cot \theta \ll$ $\delta^{-1}$, which corresponds to neglecting the component of gravity in the $y$ direction. To retain the effects of surface and interfacial tension, and tension gradients, in the longwave limit, we adopt the scalings for the capillary and Marangoni numbers,

$$
C a_{j}=\delta^{3} C_{j}, \quad M a_{j}=\delta^{2} M_{j}
$$

for $j=1,2,3$, where the $C_{j}$ and $M_{j}$ are all $O(1)$ constants. We note that with this scaling for the capillary number, we will ultimately derive a system of equations which disregard inertia, and this is consistent with the Stokes approximation made in the previous section. An alternative scaling, $\mathrm{Ca}=O\left(\delta^{2}\right)$, is also possible, and working from the Navier-Stokes equation as a starting point, will yield a generalisation of the classical Benneytype model for single-layer flow ([36]). This latter scaling was adopted by Boutounet et al. [20] for two-layer flow, working in the absence of surfactant.

Substituting the expansions (20) into the nondimensionalised form of the Stokes equations (1), at leading order we obtain

$$
0=-P_{j \xi}+m_{j} U_{j y y}, \quad P_{j y}=0, \quad U_{j \xi}+V_{j y}=0 .
$$

The equation of state (4) yields the leading order relations $\gamma_{j \xi}=-\delta^{2} M_{j} \Gamma_{j \xi}$. The normal components of the stress balances $(2)$ reduce to

$$
\begin{aligned}
P_{1}-P_{2}+\xi\left(1-R_{2}\right) & =-\frac{f_{1 \xi \xi}}{C_{1}} \\
P_{2}-P_{3}+\xi\left(R_{2}-R_{3}\right) & =-m_{2} \frac{f_{2 \xi \xi}}{C_{2}} \\
P_{3}+\xi R_{3} & =-m_{3} \frac{f_{3 \xi \xi}}{C_{3}}
\end{aligned}
$$

at $y=f_{1}, f_{2}, f_{3}$ respectively, and the tangential stress components become

$$
\begin{aligned}
m_{2} U_{2 y}-U_{1 y} & =\frac{M_{1} \Gamma_{1 \xi}}{C_{1}} \\
\frac{m_{3}}{m_{2}} U_{3 y}-U_{2 y} & =\frac{M_{2} \Gamma_{2 \xi}}{C_{2}} \\
-U_{3 y} & =\frac{M_{3} \Gamma_{3 \xi}}{C_{3}}
\end{aligned}
$$

at $y=f_{1}, f_{2}, f_{3}$ respectively. The no-slip and tangential flow conditions at the wall $y=0$ are

$$
U_{1}=0, \quad V_{1}=0 .
$$

Continuity of velocity at the two fluid interfaces requires

$$
\begin{array}{ll}
U_{1}=U_{2}, & V_{1}=V_{2} \\
U_{2}=U_{3}, & V_{2}=V_{3}
\end{array}
$$

at $y=f_{1}, f_{2}$ respectively, and the kinematic condition at the free surface requires

$$
f_{j \tau}+U_{j} f_{j \xi}=V_{j}
$$

at $y=f_{3}$.

Henceforth we assume equal density fluids so that $R_{2}=R_{3}=1$. Solving the momentum equations (22) and satisfying the boundary conditions (23)-(32), we derive the long-wave evolution equations

$$
f_{j \tau}+q_{j \xi}=0,
$$

for $j=1,2,3$, where the fluxes $q_{j}$ are given by

$$
\begin{gathered}
q_{1}=\int_{0}^{f_{1}} U_{1} \mathrm{~d} y, \quad q_{2}=q_{1}+\int_{f_{1}}^{f_{2}} U_{2} \mathrm{~d} y \\
q_{3}=q_{2}+\int_{f_{2}}^{f_{3}} U_{3} \mathrm{~d} y
\end{gathered}
$$

and the velocities are

$$
U_{j}=\frac{1}{2 m_{j}} y^{2} P_{j \xi}+A_{j} y+B_{j}, \quad j=1,2,3,
$$

with

$$
\begin{aligned}
& A_{1}=-\frac{M_{1} \Gamma_{1 \xi}}{C_{1}}-f_{1} P_{1 \xi}+f_{1} P_{2 \xi}+m_{2} A_{2}, \\
& A_{2}=-\frac{M_{2} \Gamma_{2 \xi}}{C_{2}}-\frac{f_{2} P_{2 \xi}}{m_{2}}+\frac{f_{2} P_{3 \xi}}{m_{2}}+\frac{m_{3} A_{3}}{m_{2}}, \\
& A_{3}=-\frac{M_{3} \Gamma_{3 \xi}}{C_{3}}-\frac{f_{3} P_{3 \xi}}{m_{3}},
\end{aligned}
$$

and $B_{1}=0$ and

$$
\begin{aligned}
& B_{2}=\frac{f_{1}^{2}}{2}\left(P_{1 \xi}-\frac{P_{2 \xi}}{m_{2}}\right)+f_{1}\left(A_{1}-A_{2}\right), \\
& B_{3}=\frac{f_{2}^{2}}{2}\left(\frac{P_{2 \xi}}{m_{2}}-\frac{P_{3 \xi}}{m_{3}}\right)+f_{2}\left(A_{2}-A_{3}\right)+B_{2},
\end{aligned}
$$

where the pressures are given by

$$
\begin{aligned}
& P_{1}=-\xi-\frac{f_{1 \xi \xi}}{C_{1}}-m_{2} \frac{f_{2 \xi \xi}}{C_{2}}-m_{3} \frac{f_{3 \xi \xi}}{C_{3}}, \\
& P_{2}=-R_{2} \xi-m_{2} \frac{f_{2 \xi \xi}}{C_{2}}-m_{3} \frac{f_{3 \xi \xi}}{C_{3}}, \\
& P_{3}=-R_{3} \xi-m_{3} \frac{f_{3 \xi \xi}}{C_{3}} .
\end{aligned}
$$

The surfactant transport equations (19) reduce to (assuming negligible diffusion),

$$
\Gamma_{j \tau}+\left(\Gamma_{j} U_{j}\right)_{\xi}=P e_{j}^{-1} \Gamma_{j \xi \xi},
$$

where $U_{j}$ is evaluated at $y=f_{j}$. The task now is to solve the coupled nonlinear equations (33) and (44) to 
determine the interface and free surface shapes and to find the surfactant concentrations. Henceforth, and unless otherwise stated, we will assume infinite surfactant Péclet numbers and set the right hand sides of (44) to zero.

In the basic state, the interfaces and free surface are flat and the surfactant concentrations are uniform. To compute linearised growth rates for small perturbations, we expand about the base states by writing

$$
\begin{gathered}
f_{1}=1+\epsilon \tilde{\alpha}_{1} \mathrm{e}^{\mathrm{i} k(\xi-c \tau)}, \quad f_{j}=\left(1+r_{j}\right)+\epsilon \tilde{\alpha}_{j} \mathrm{e}^{\mathrm{i} k(\xi-c \tau)}, \\
\Gamma_{j}=1+\epsilon \tilde{\Gamma}_{j} \mathrm{e}^{\mathrm{i} k(\xi-c \tau)},
\end{gathered}
$$

where $j=2,3$ and $\epsilon \ll 1$. The constant amplitudes $\tilde{\alpha}_{j}, \tilde{\Gamma}_{j}$ are to be found and the addition of the complex conjugate to the perturbation terms is understood. Substituting into the governing equations (33)-(44) we derive a polynomial equation for the complex wavespeed $c$. For three-layer flow the polynomial is of sixth order meaning that there are in general six normal mode solutions for each choice of the perturbation wave number $k$. The system of equations for two layer flow is obtained from (33)-(44) simply by setting $A_{3}=B_{3}=P_{3}=0$. In this case, we obtain a fourth order polynomial from the linear stability analysis producing four normal modes for each $k$. For both two-layer and three-layer flow the coefficients of the polynomial are lengthy algebraic expressions which we suppress in the interest of brevity. As a check on the calculation, we have confirmed that the presently computed growth rates coincide with those obtained using the theory for Stokes flow in section II on taking the limit $k \rightarrow 0$ and on adopting the scalings (21).

The main focus of this section is on computing travelling wave solutions to the long-wave system. These are expected to emerge as Hopf bifurcations from the uniform, equal-layer-thickness state at the neutral stability point where the linear growth rate vanishes. To seek such solutions, we introduce the travelling-wave coordinate $z=\xi-c_{w} \tau$ and write $f_{j}=\tilde{f}_{j}(z), \Gamma_{j}=\tilde{g}_{j}(z)$ and $U_{j}=\tilde{U}_{j}(y, z), q_{j}=\tilde{q}_{j}(z)$, where $c_{w}$ is the wave speed to be found. Substituting into (33) and (44), and integrating once, we obtain

$$
-c_{w} \tilde{f}_{j}+\tilde{q}_{j}=d_{j}, \quad\left(\tilde{U}_{j}-c_{w}\right) \tilde{g}_{j}=e_{j},
$$

where the $d_{j}$ and $e_{j}$ are constants of integration, and $U_{j}$ is evaluated at $y=\tilde{f}_{j}$. Assuming periodic waves of period $L$, we express the surface profiles in the form of the Fourier series

$$
\tilde{f}_{j}=\sum_{n=-\infty}^{\infty} \tilde{f}_{j}^{(n)} \mathrm{e}^{\mathrm{i} n k z},
$$

where $k=2 \pi / L$, with analogous expressions for the other variables. Substituting into the remaining equations in (33)-(44) we obtain expressions for the flux coefficients $\tilde{q}_{j}^{(n)}$. Truncating the Fourier series (47) at the finite level $n=N$, for $M$ fluid layers (46) yields a system of $2 M(2 N+1)$ algebraic equations for the
$2 M(2 N+1)+(2 M+1)$ unknowns $\tilde{f}_{j}^{(n)}, \tilde{g}_{j}^{(n)}, d_{j}, e_{j}$ (for $j=1, \ldots M)$, and $c_{w}$. A further $2 M$ equations are provided by fixing the mean layer thicknesses and the mean surfactant concentrations, specifically demanding that

$$
\tilde{f}_{1}^{(0)}=1, \quad \tilde{f}_{j}^{(0)}=1+\sum_{i=2}^{j} r_{i}, \quad \tilde{g}_{j}^{(0)}=1,
$$

for $j=2, \ldots M$. Enforcing these conditions corresponds to fixing the fluid volume within each flow period.

A suitable condition to break the translational invariance of the travelling-wave solutions gives the final equation required to close the system. We impose that

$$
\int_{0}^{L} z\left(\tilde{f}_{1}(z)-1\right) \mathrm{d} z=0
$$

which requires $\sum_{n=-N, n \neq 0}^{N} \tilde{f}_{1}^{(n)} / n=0$.

The computations are performed efficiently in Matlab making use of the fast Fourier transform facility to compute the Fourier coefficients and Newton's method to solve the nonlinear system of algebraic equations. To lock onto a particular solution branch, we take as the initial guess for the Newton iterations the normal mode properties determined from the linear stability calculation (see 45) close to the neutral point where the imaginary part of the complex wavespeed $c$ is almost zero. Once a solution on the travelling-wave branch has been found, the remainder of the branch can be traced out using continuation methods. In the following sections, parameter sets are chosen to highlight the different types of solution behaviour which are encountered in two-layer and threelayer systems. In particular we select parameter values to obtain one or more neutral stability points from which travelling wave branches emerge via a Hopf bifurcation. For a three-layer flow for example, as discussed above, we have identified at most three unstable modes for a given parameter set, and so we expect to find at most three travelling wave branches. The number of Fourier modes required to accurately resolve the travelling wave profiles varies, and we typically take from $N=20$, but a considerably larger value may be required for profiles with more intricate features. Some comment on physical parameter values for typical fluids that might be used in experiment will be made in section VI.

\section{A. Two-layer flow}

The linear stability graphs we compute for two layers are qualitatively similar to those found under conditions of Stokes flow in section II. This is illustrated by the example two-layer case shown in figure 7 . We observe that the general character of the neutral curves shown in this figure are broadly in line with those computed for Stokes flow in section III; for example, both feature an island of unstable modes when surfactant is present on the free surface (see figure 2(b) for the Stokes case). 
$(a)$

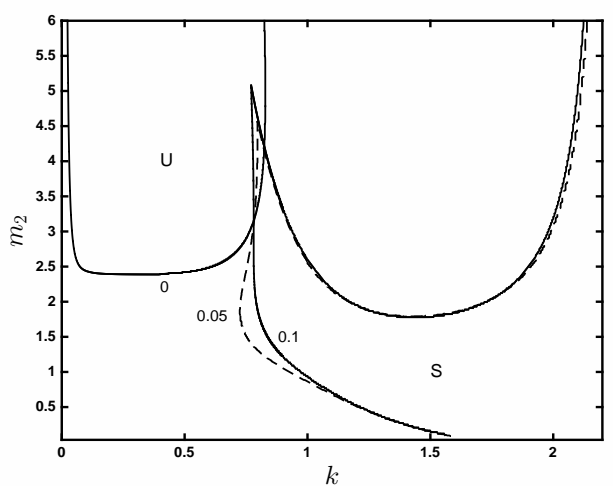

(b)

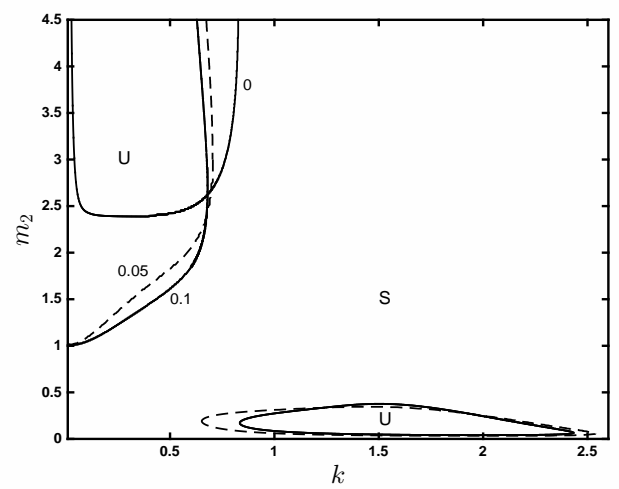

FIG. 7. Long-wave neutral curves for two-layer flow with $C_{1}=2.5, C_{2}=1, r_{2}=0.5, R_{2}=1:(a) M a_{2}=0$ and various $M a_{1}$; (b) $M a_{1}=0$ and various $M a_{2}$. Regions are marked stable $(\mathrm{S})$ and unstable $(\mathrm{U})$.

The upper panel of figure $8(a)$ shows the stability graph for an unstable clean system with $m_{2}=5$. We note that the eigenmodes associated with the instability in this figure have properties consistent with those required to produce stability or instability from a physical standpoint, as discussed by Gao and $\mathrm{Lu}$ [19]. For example, at $k=0.1$, we find the two growth rates are $s=2.8 \times 10^{-5}$ and $s=-1.3 \times 10^{-3}$. The corresponding perturbations in (45) have the properties $\arg \left(\tilde{\alpha}_{2} / \tilde{\alpha}_{1}\right)=-0.998$ and $\arg \left(\tilde{\alpha}_{2} / \tilde{\alpha}_{1}\right)=6 \times 10^{-4}$ for the unstable and stable mode respectively. These are very close to the phase shifts of $\pi$ and 0 respectively identified for unstable/stable modes by Gao and Lu (see their paper for further discussion of the physical mechanisms at work).

Henceforth, we focus on travelling-wave solutions to the long-wave system. The lower panel of figure $8(a)$ shows the travelling wave branch which emanates from the neutral stability point at $k=0.765$. The branch is characterised by the interfacial wave height $\mathcal{A}=$ $\max \left(f_{1}\right)-\min \left(f_{1}\right)$. As the wave number $k$ is lowered, the travelling-wave solutions approach a pulse-like state with the disturbance on each surface localised in space (a)
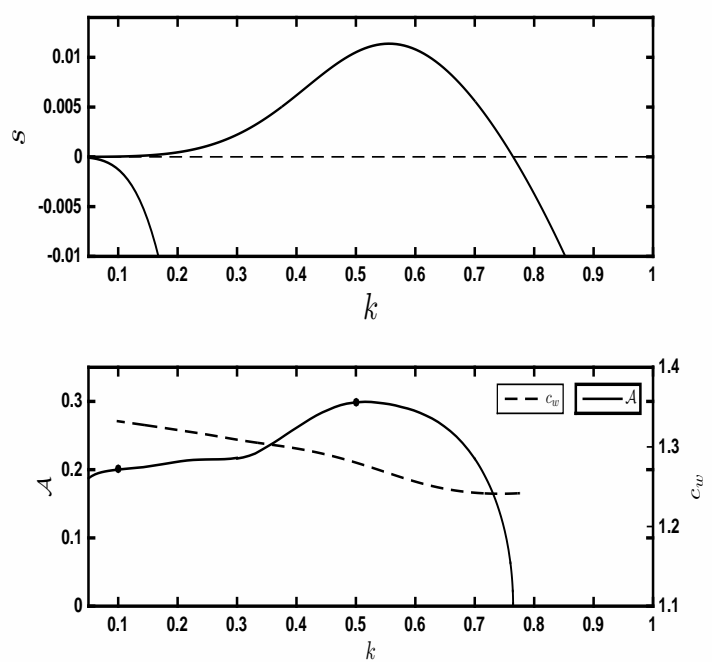

(b)
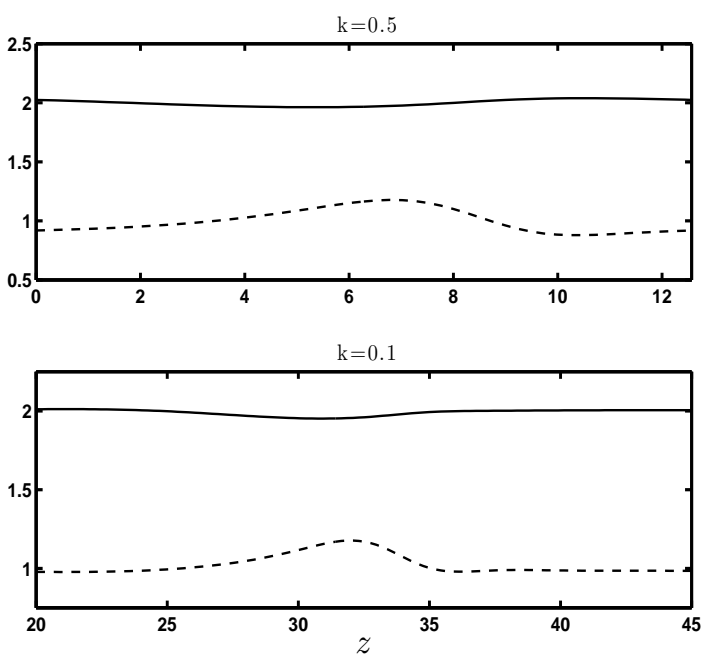

FIG. 8. Clean two-layer long-wave flow with $C a_{1}=1$, $C a_{2}=1, r_{2}=1, m_{2}=5, R_{2}=1, M a_{1}=0$ and $M a_{2}=0:(a)$ Growth rates $s=\operatorname{Im}(k c)$ for two-layer long-wave system (upper panel) and travelling-wave interface height $\mathcal{A}$ and wave speed $c_{w}$ (lower panel); (b) Film profiles at $k=0.5$ (upper panel) and $k=0.1$ (lower panel), indicated by dots in $(a)$.

and the film surfaces flat in the far-field. Sample profiles at $k=0.5$ and $k=0.1$ are shown in figure $8(b)$.

The upper panel of figure 9 shows the growth rate $s \equiv \operatorname{Im}(k c)$ plotted against wave number for an unstable two-layer flow with equal viscosity fluids, equal layer thicknesses and with surfactant on both the interface and the free surface. The instability is attributed to the presence of surfactant since the same flow is stable when $M a_{1}=M a_{2}=0 . \quad$ A travelling-wave branch emerges from the neutral point at $k=1.03$ where the growth rate of the unstable mode vanishes (middle panel of figure 9 ). 

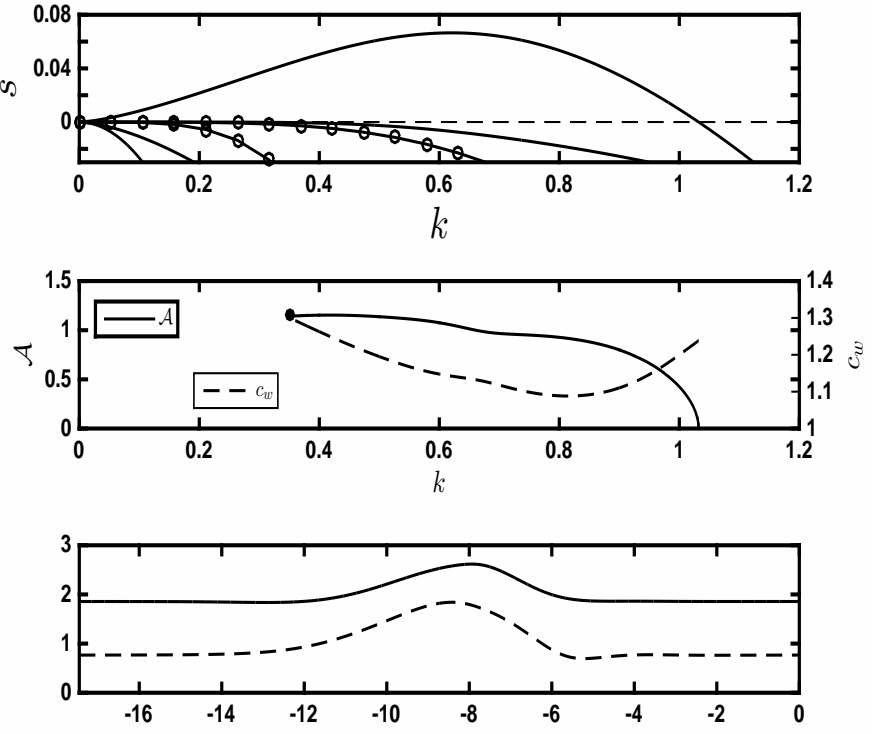

FIG. 9. Two-layer long-wave flow with $C a_{1}=1.1, C a_{2}=$ $1, r_{2}=1, m_{2}=1, R_{2}=1, M a_{1}=M a_{2}=1$. Upper panel: Growth rates $s=\operatorname{Im}(k c)$ for the two-layer long-wave system. The lines with circles show the two growth rates for no surfactant, $M a_{1}=M a_{2}=0$. Middle panel: travellingwave height $\mathcal{A}$ and wave speed $c_{w}$. Lower panel: Free surface and interface profiles at $k=0.35$, indicated by the dot in the middle panel.
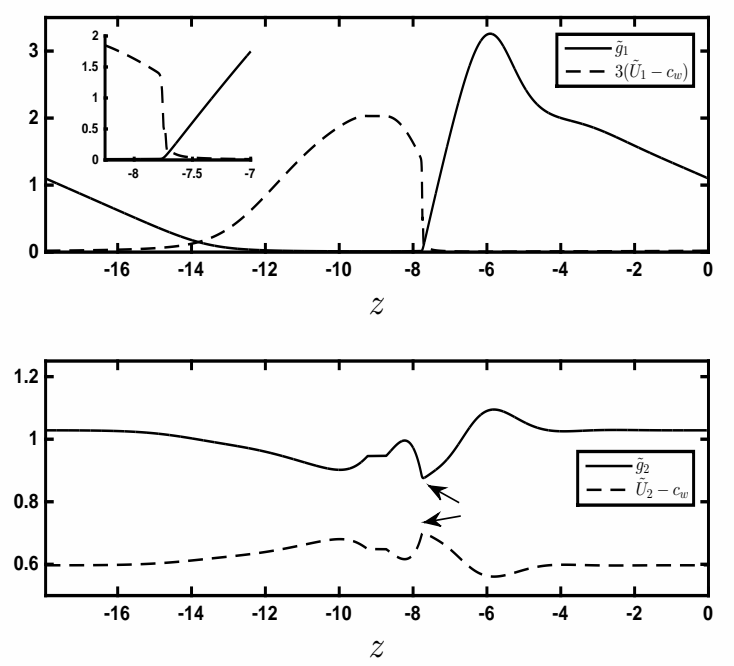

FIG. 10. Surfactant and interfacial velocity profiles for figure 9 at $k=0.35$. Upper panel $\tilde{g}_{1}$ and shifted interfacial velocity $\tilde{U}_{1}-c_{w}$. The inset shows a close-up near the shock at $z_{s} \approx$ -7.8. Lower panel: $\tilde{g}_{2}$ and shifted interfacial velocity $\tilde{U}_{2}-c_{w}$. The arrows indicate regions of very rapid change in gradient at $z=z_{s}$. The profiles were computed using $N=1400$ Fourier modes.
As in the previous clean example, the film profiles approach pulse states as the travelling-wave branch is followed from the bifurcation point at $k=1.03$. These are shown in the lowermost panel of figure 9 when $k=0.35$. The pulses are phase-locked and travel together at the same speed, $c_{w}=1.32$. As the solution branch is followed from the bifurcation point, the interfacial surfactant concentration eventually touches zero, and as $k$ is decreased further there widens out an almost clean region in which $\tilde{g}_{1}$ is very small. This region is clearly seen in the surfactant profiles at $k=0.35$ shown in figure $9(\mathrm{~b})$, where the interfacial surfactant level is almost zero over part of the wave period. Evidently a shock is emerging at $z=z_{s} \approx-7.8$ where there is a rapid change in gradient. This is expected to sharpen into a true shock as $k \rightarrow 0$. The regions of rapid change require a large number of Fourier modes to resolve accurately (we took $N=3500$ to compute the profiles shown in figure 10). However, it is clear from the computations that the constant $e_{1}$ in (46) approaches zero as $k \rightarrow 0$. In the limit of small $k$, then, we expect the balance on the interface,

$$
\left(\tilde{U}_{1}-c_{w}\right) \tilde{g}_{1}=0
$$

Accordingly, we may construct a solution consisting of clean regions, where $\tilde{g}_{1}=0$, and regions in which the interfacial velocity matches the wave speed $\tilde{U}_{1}=c_{w}$. In the case shown in figure 10 , these regions are separated by a shock with a discontinuity in the velocity and in the slope of the surfactant concentration. The near-clean region occupies approximately the zone $-12<z<-7.8$. There is a smooth transition into the region from the left but a sharp transition at the near-shock at $z=z_{s} \approx$ -7.8. The surfactant profile on the free surface is shown in the lower panel of the figure together with the surface velocity. In this case, the computations show that the constant $e_{2}$ in (46) approaches a non-zero value as $k \rightarrow 0$. The gradient in both the surfactant and velocity profiles jumps sharply at $z=z_{s}$, which is consistent with the relation $\left[\left(\tilde{U}_{2}-c_{w}\right) \tilde{g}_{2}\right]_{\xi}=0$ obtained by taking the first derivative of (46).

To investigate the effect of surfactant diffusivity, we repeated the calculations for the same parameter values as figure 9 but with the non-zero Péclet numbers $P e_{1}=P e_{2}=0.001$. Very similar results were found with shock-type behaviour again appearing in the interfacial surfactant profile. It might alternatively be supposed that the shock-development and the appearance of a near- clean region is the result of using a linear relationship for the equation of state (4). We repeated the calculation for figure 9 instead using the nonlinear Langmuir equation of state (see, for example, Pozrikidis [33], p. 234), and found that when the travelling-wave branch is followed from the bifurcation point, a similar state is reached with an extended region which is almost devoid of surfactant.

The results in section III indicate that at most two of the possible four normal modes can be unstable across the range of wave numbers. The upper panel of Figure 
$(a)$
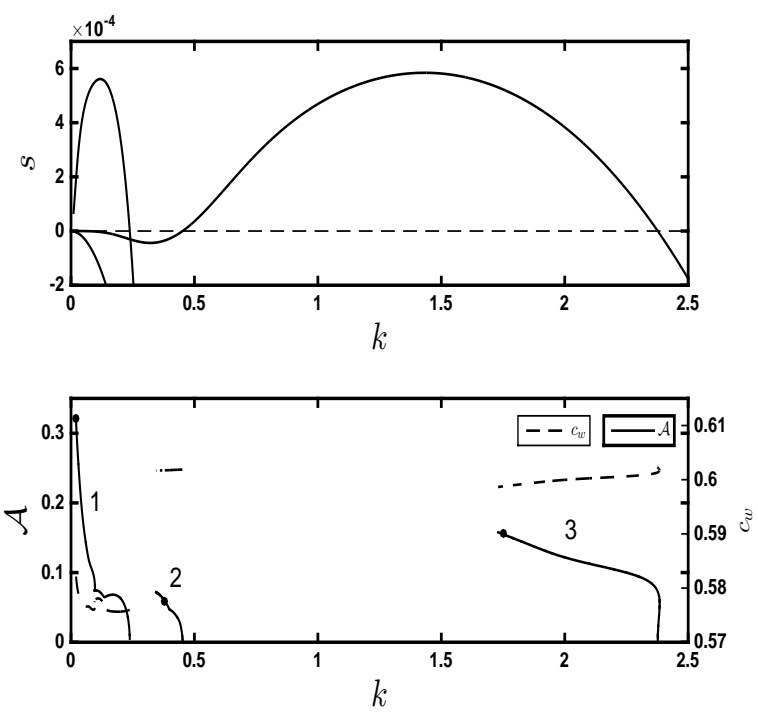

(b)
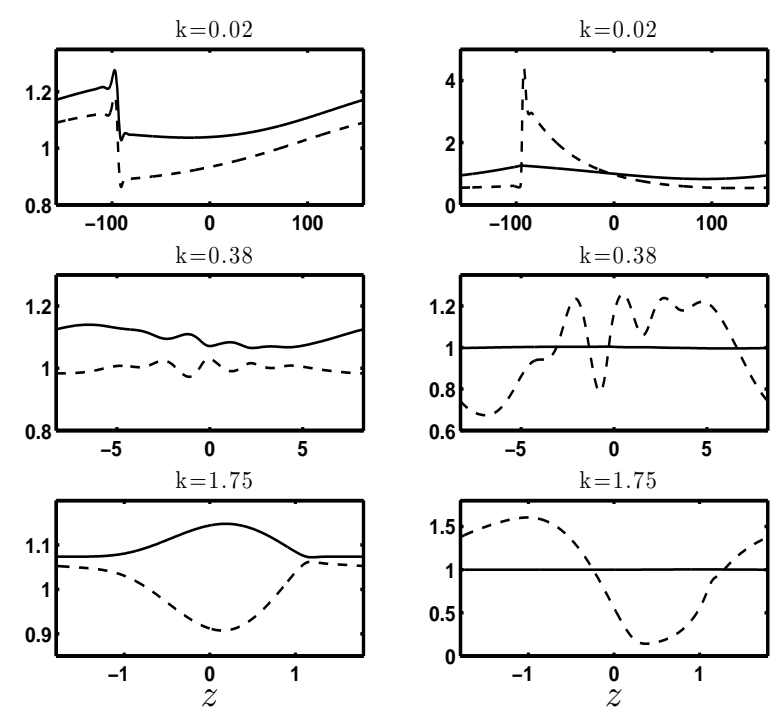

FIG. 11. Two-layer long-wave flow with $C a_{1}=1, C a_{2}=1$, $r_{2}=0.1, m_{2}=2, R_{2}=1, M a_{1}=0.1$ and $M a_{2}=10.391$ : (a) Growth rates $s=\operatorname{Im}(k c)$ for two-layer long-wave system (upper panel) and interface wave height $\mathcal{A}$ and wave speed $c_{w}$ (lower panel); (b) Film (left) and surfactant (right) travellingwave profiles at $k=0.02, k=0.38$ and $k=1.75$, on branches $1,2,3$ in (a) respectively, indicated by dots on these branches. Interface profiles are shown with broken lines and surface profiles with solid lines.

11(a) shows the linear growth rates plotted against wave number for the case $C a_{1}=1, C a_{2}=1, r_{2}=0.1, m_{2}=2$, $R_{2}=1, M a_{1}=0.1$ and $M a_{2}=10.391$. In this case the upper layer is more viscous than the lower layer and the flow is linearly unstable even in the absence of surfactant. For this case, two of the four modes are unstable over the wave number range. The presence of a window of stable $(a)$
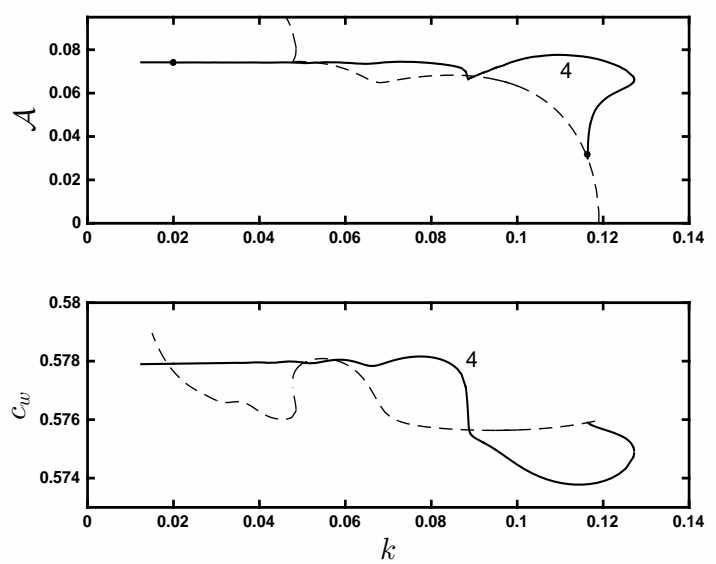

(b)
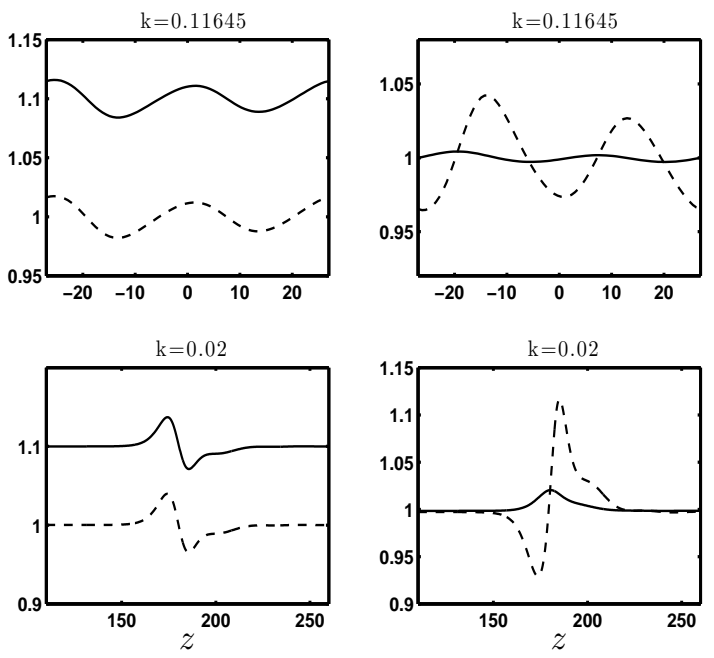

FIG. 12. Same conditions as for figure 11. (a) Wave height $\mathcal{A}$ and wave speed $c_{w}$ on the bifurcation branch (solid line) emerging subharmonically from branch 1 (broken line) in figure 11(a). (b) Film (left) and surfactant (right) profiles at $k=0.11645$ and $k=0.02$ indicated by dots in $(a)$. Interface profiles are shown with broken lines and surface profiles with solid lines.

wave numbers between the two unstable ranges means that there are three neutral stability points (at $k=0.238$, $k=0.452$ and $k=2.377$ ) and we therefore expect to find three travelling-wave solution branches emanating from these. The wave branches are delineated in the lower panel of figure 11(a). The wave speed $c_{w}$ is indicated in the figure and remains almost constant along each of the branches. Sample film and surfactant profiles on each of these branches are shown in figure 11(b). As in the previous case the interfacial surfactant profile develops a steep gradient while the free surface surfactant concentration suffers only a minor deviation from its mean level. The branches stemming from $k=0.238$ and $k=0.452$ were not traced further than shown because of computational 
difficulties. As branch 3 is followed from the bifurcation point at $k=2.377$ to smaller wavenumber, eventually the interfacial surfactant profile touches down to zero. Simultaneously the interface and the free surface almost touch, producing a travelling-wave state with periodic bulges of upper layer fluid. On branch 1 , for small $k$ both the film profiles and the surfactant concentration profiles exhibit regions of relatively rapid variation. The film profiles in this region have a small ridge immediately before the descent in the surface level and as such are reminiscent of the capillary-ridge profiles found in film flows in other contexts (e.g. [37, 38]).

For the same parameter values, we also identified a fourth branch which appears as a subharmonic bifurcation from branch 1 . This branch is shown as a solid line in figure $12(a)$; the broken line in the same figure corresponds to branch 1 from figure $11(a)$, but with two wave periods included in the range $[0,2 \pi / k]$. Wave profiles close to the point at which the solid line bifurcates from the broken line are almost $\pi / k$ periodic, as can be seen for the case $k=0.11645$ shown in the upper panels of figure $12(b)$. Following the bifurcated branch to smaller wavenumber, the profiles are seen to develop into pulses. The solution for $k=0.02$ is shown in the lower panels of figure $12(b)$. The propagation speed of the pulses is $c_{w}=0.58$. It is notable that for these pulse solutions the corresponding surfactant profiles do not develop clean patches as was found for the example in figure 9. Further computations showing travelling waves for other parameter sets can be found in Thompson [39].

As has already been noted, the physical mechanisms underlying the instability of clean two-layer flows have been discussed recently by Gao and Lu [19], following earlier work by Jiang et al. [18]. In particular Gao and $\mathrm{Lu}$ provided an explanation for the instability when the upper layer is more viscous than the lower layer with reference to the disturbance flows generated at the free surface and at the interface. A discussion of the physical mechanisms responsible for Marangoni-driven instability in sheared film flow has been presented by Wei [40]. A partial physical explanation for the saturated travellingwave profiles described in this section may be provided as follows. First, we take an example of a clean flow and focus on the case of a travelling pulse pair shown in figure 8(b). According to Gao and $\mathrm{Lu}$ [19] the pulselike disturbances generate disturbance flows $\mathbf{u}^{s}$ and $\mathbf{u}^{c}$, resulting from $(\mathrm{s})$ the shear stress condition at the free surface and (c) the velocity continuity condition at the interface. We viewing the flow in a reference frame travelling at the wave speed $c_{w}$, and note that $c_{w}>1$ (see figure 8a) so that the pulses are travelling more rapidly than the surface velocity of the flat state further upstream and downstream (we recall that velocities have been non-dimensionalised using the surface velocity as the reference scale). Accordingly, if the wave is travelling to the right, the prevailing flow inside the layers in this frame is from right to left. When $m>1$, the disturbance flows $\mathbf{u}^{s}$ and $\mathbf{u}^{c}$ are both directed from left to right (see Gao and $\mathrm{Lu}$ [19] figure 3), and against the prevailing flow direction. Therefore these flows decelerate the fluid beneath the pulses, thereby sustaining the disturbance.

\section{B. Three-layer flow}

A sample case for clean three-layer flow is shown in figure 13. The system has a more viscous layer between two layers of equal viscosity, and is linearly unstable. The stability graph is shown in the upper panel of figure 13(a). A travelling wave branch emerges from the neutral point at $k=0.813$. Following the branch, we find that the travelling wave profiles approach a state with localised, but slowly-varying, pulses on each of the film surfaces. Profiles at $k=0.5$ and $k=0.05$ are shown in $13(b)$. The speed of propagation of the bumps at $k=0.05$ is $c_{w}=2.36$.

Figure 14 shows a configuration with surfactant on all three of the film surfaces. There are three unstable modes, as can be seen in the stability graph in the upper panel of figure 14(a). Travelling wave branches emerge from the neutral points at $k=0.752, k=1.196$ and $k=1.373$, and are shown in the lower panel. Typical film and surfactant profiles are shown in figure $14(b)$. It is noticeable that in the profiles for $k=1.35$ the free surface and interface 2 almost touch, and for $k=1.6$ the two interfaces almost touch. The profiles at $k=0.3$ have a pulse-like structure, and the surfactant profile is evidently developing a shock in the gradient and a nearclean region, very much like the case studied for two-layer flow in figures 9 and 10. Multiple travelling wave solutions exist for some values of $k$. For example, there are three solutions in the range $1.35<k<1.373$.

Figure 15 shows results for almost the same parameter set as figure 14 but for a clean free-surface and clean interface 1 . There is only one unstable mode and a single travelling wave branch emerging from the neutral point at $k=0.700$. The film profiles develop into pulses as the travelling wave branch is followed to smaller wavenumber. Similar to the two-layer case shown in figure $9(b)$ the pulses are phase-locked and travel together as a group (for the profiles shown at $k=0.22$ the propagation speed is $\left.c_{w}=3.82\right)$.

Next we demonstrate the influence of surfactant on the speed of the travelling waves. Figure 16(a) shows the effect of varying any one of the Marangoni numbers for the parameter set studied in the three-layer flow case of figure 15. The solid and dashed curves terminate where two of the interfaces come into close contact. The general trend found here, and which we also find for the other examples presented in this section, is that surfactant tends to lower the wave speed below that found for an entirely clean system (although it may also raise it, as shown by the dot-dashed curve in figure 16a). This example also provides a qualitatively different feature in the wave profiles to those seen before. Figure 16(b) shows that the two interfaces are almost touching and the surfactant on 
$(a)$
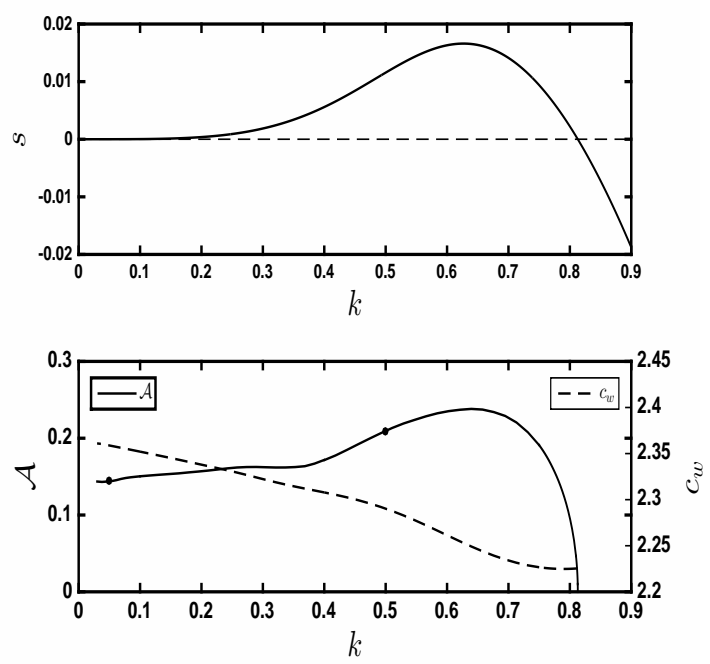

(b)
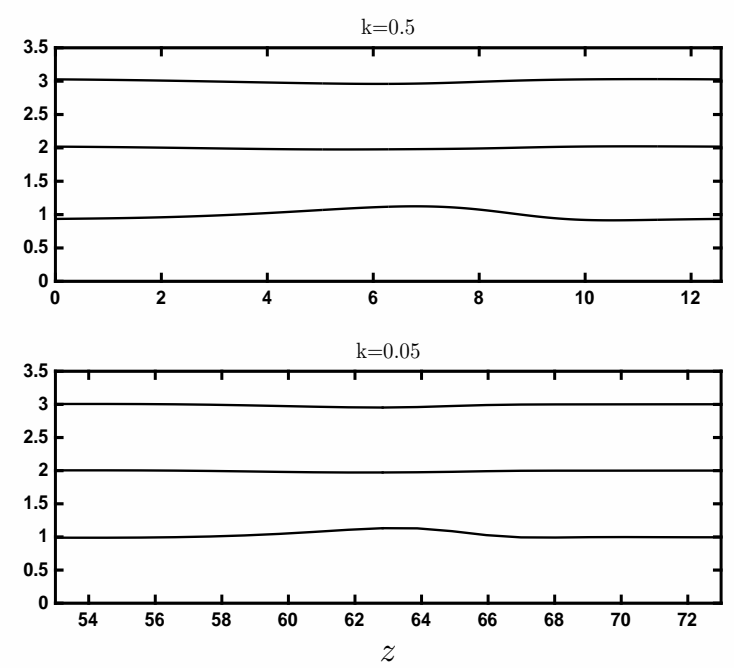

FIG. 13. Clean three-layer long-wave flow $C a_{1}=C a_{2}=$ $C a_{3}=1, r_{2}=r_{3}=1, m_{2}=2, m_{3}=1, R_{2}=R_{3}=1$, $M a_{1}=M a_{2}=M a_{3}=0:(a)$ Growth rates $s=\operatorname{Im}(k c)$ for the three-layer long-wave system (upper panel) and travellingwave lower interface height $\mathcal{A}=\max \left(f_{1}\right)-\min \left(f_{1}\right)$ and wave speed $c_{w}$ (lower panel); (b) Film profiles at $k=0.5$ (upper panel) and $k=0.05$ (lower panel), indicated by dots in $(a)$.

the lowermost interface occupies a compact and very narrow region in an almost spiked distribution. This region narrows further and the height of the spike increases, and the two interfaces come closer together, when $k$ is reduced further. This contrasts with the behaviour seen in figure 14(b), where two interfaces come into near contact without an apparently singular response in the surfactant concentration. In the present case, the singular behaviour is attributed to the fact that the local velocity on the lowermost interface approaches the value of the wave speed $c_{w}$. In the limit this requires the surfactant (a)
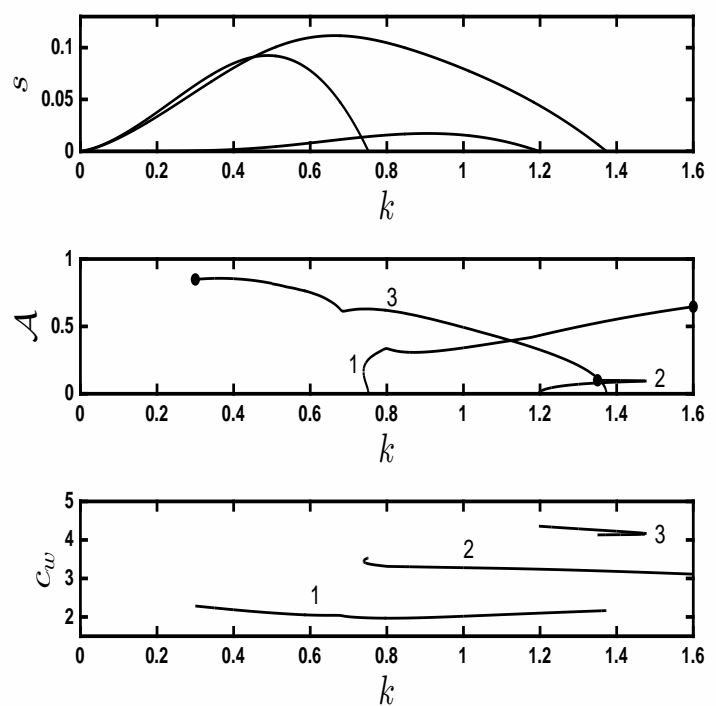

(b)
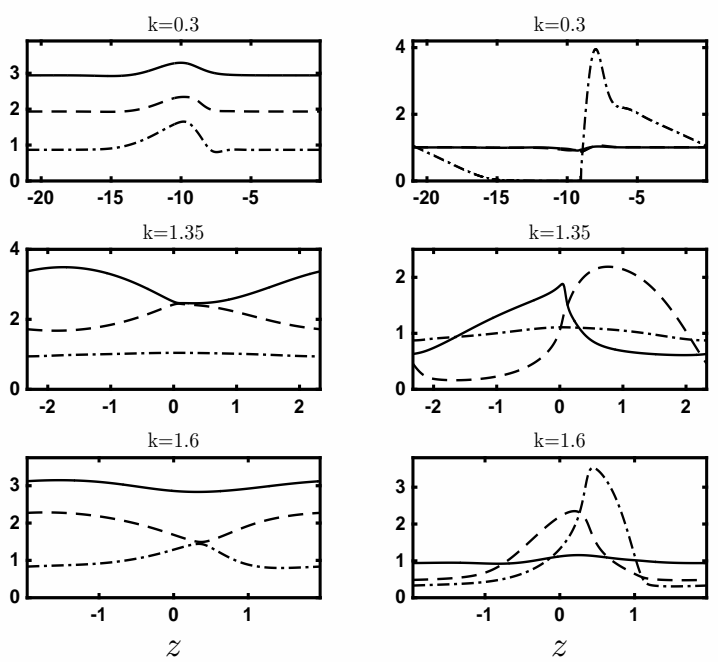

FIG. 14. Three-layer long-wave flow $C a_{1}=1.5, C a_{2}=1$, $r_{2}=r_{3}=1, m_{2}=m_{3}=1, R_{2}=R_{3}=1, M a_{1}=$ $M a_{2}=M a_{3}=1:$ (a) Growth rates $s=\operatorname{Im}(k c)$ for the three-layer long-wave system (upper panel) and travellingwave lower interface height $\mathcal{A}=\max \left(f_{1}\right)-\min \left(f_{1}\right)$ (middle panel) and wave speed $c_{w}$ (lower panel); (b) Film (left) and surfactant (right) travelling-wave profiles on branches 1, 2, 3 in $(a)$ respectively at $k=1.6,1.35$ and 0.3 , indicated by dots in $(a)$. Lower/upper interface profiles are shown with dotdashed/dashed lines and free surface profiles are shown with solid lines.

concentration on the lowermost interface to grow without bound in order to maintain a balance in (46). 
$(a)$
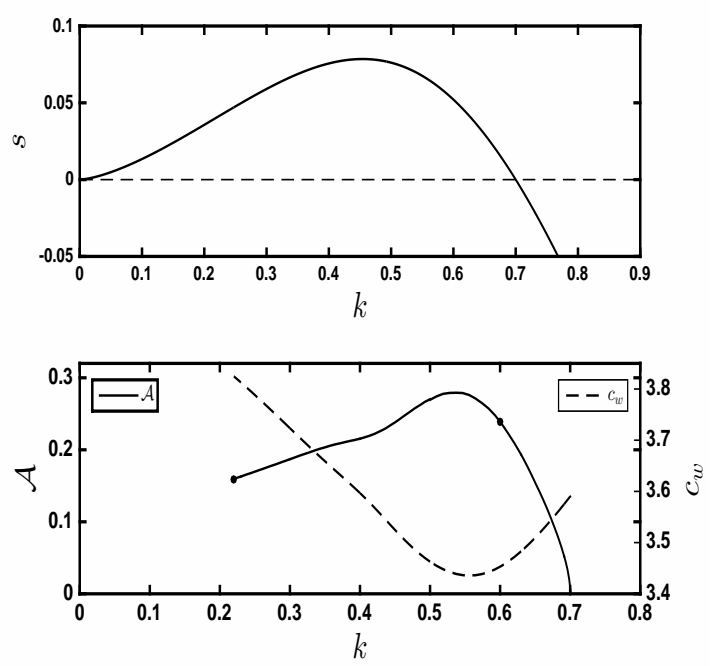

(b)
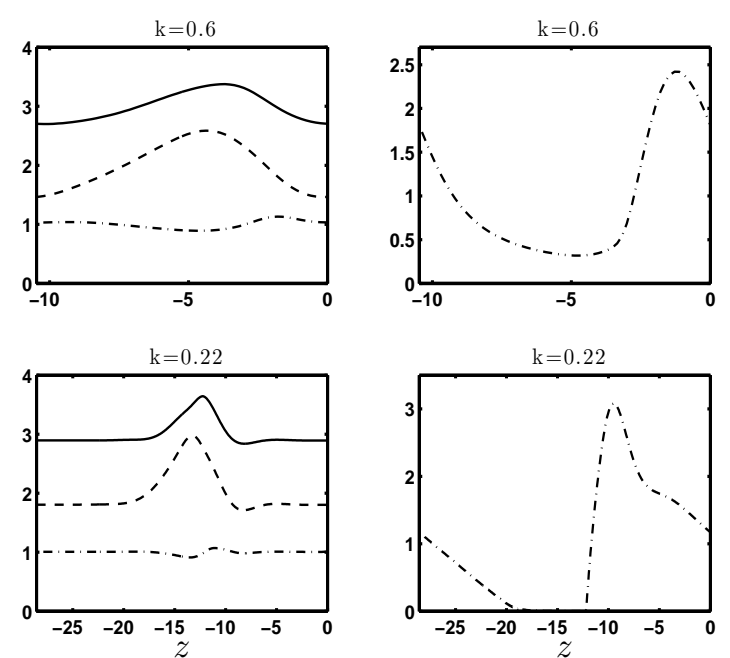

FIG. 15. Three-layer long-wave flow $C a_{1}=1.5, C a_{2}=1$, $r_{2}=r_{3}=1, m_{2}=m_{3}=1, R_{2}=R_{3}=1, M a_{1}=0$, $M a_{2}=1.0, M a_{3}=0:(a)$ Growth rates $s=\operatorname{Im}(k c)$ for the three-layer long-wave system (upper panel) and travellingwave lower interface height $\mathcal{A}=\max \left(f_{1}\right)-\min \left(f_{1}\right)$ and wave speed $c_{w}$ (lower panel); (b) Film (left) and surfactant (right) travelling-wave profiles at $k=0.6$ and $k=0.22$, indicated by dots in $(a)$. Lower/upper interface profiles are shown with dot-dashed/dashed lines and free surface profiles are shown with solid lines.

\section{Time-dependent simulations}

Having described the existence of a range of travelling wave phenomena in the previous sections, it is of interest to ask what is likely to be seen in a physical experiment. To gain some insight into the issue of stability, we perform time-dependent simulations of the longwave equations. We solve (33) and (44) numerically us- $(a)$
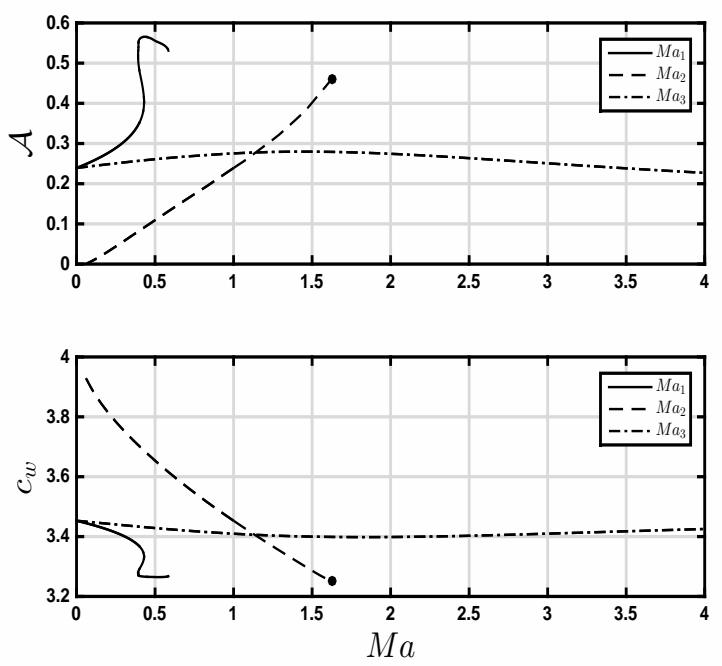

(b)
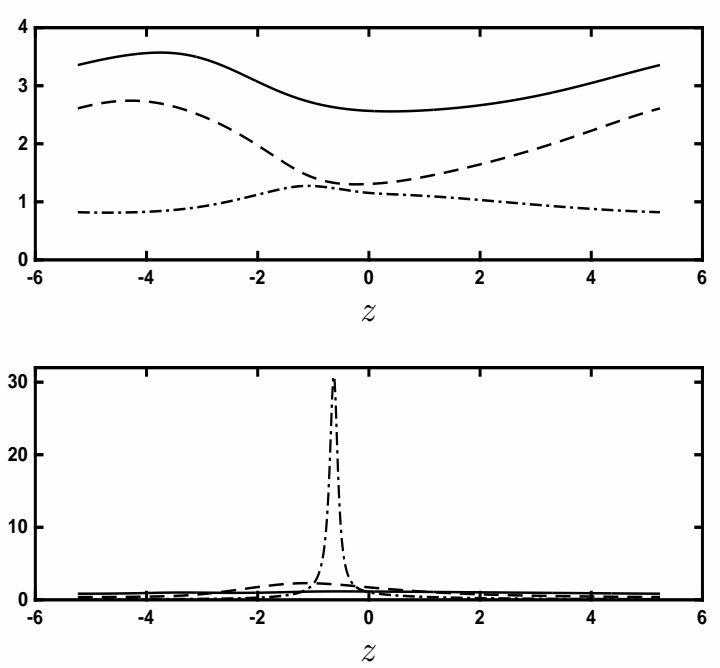

FIG. 16. Three-layer long-wave flow $C a_{1}=1.5, C a_{2}=1$, $r_{2}=r_{3}=1, m_{2}=m_{3}=1, R_{2}=R_{3}=1$ and $k=0.6$. (a) Variation of lower interface wave height $\mathcal{A}=\max \left(f_{1}\right)-$ $\min \left(f_{1}\right)$ and wave speed $c_{w}$ with Marangoni number. In each case one Marangoni number is varied and the other two are zero. (b) Wave profiles (upper panel) and surfactant profiles (lower panel) at $M a_{2}=1.629\left(M a_{1}=M a_{3}=0\right)$ corresponding to the dots in (a). Lower/upper interface profiles are shown with dot-dashed/dashed lines and free surface profiles are shown with solid lines

ing a fully implicit scheme based on Newton iterations and Fourier pseudospectral representation of the spatial derivatives on a grid of points equally-spaced in the range $0 \leq x \leq L_{c}$, for chosen domain size $L_{c}$. The scheme is second order accurate in time. The equations are integrated forward in time starting from the initial profiles 
of wavelength $L_{0}$ at $\tau=0$,

$$
\begin{aligned}
f_{1}(\xi, 0) & =1+0.2 \cos \left(2 \pi \xi / L_{0}\right), \\
f_{j}(\xi, 0) & =\left(1+r_{j}\right)+0.2 \cos \left(2 \pi \xi / L_{0}\right), \\
\Gamma_{j}(\xi, 0) & =1+0.2 \cos \left(2 \pi \xi / L_{0}\right),
\end{aligned}
$$

for $j=1,2$ or $j=1,2,3$ for two-layer/three-layer flow respectively.

We first investigate the stability of the two-layer travelling waves found for the clean system in figure 8 . We fix the computational domain size at $L_{c}=4 \pi$ and take $L_{0}=L_{c}$ in (51). The time evolution of the film wave heights $\mathcal{A}_{j}=\max \left(f_{j}\right)-\min \left(f_{j}\right)$ are shown in the upper panel of figure 17. After an initial transient, the wave heights eventually saturate and travelling waves become established. The saturated profiles are shown in the lower panel. The travelling-wave state computed in section IV A is superimposed and the two coincide almost exactly. This indicates that the travelling-waves shown in figure 8 are stable, at least to perturbations of the same wavelength. In a physical experiment, however, perturbations of wavelength larger than the wave period may be present. To investigate this, we repeated the simulation taking $L_{0}=4 \pi$ in (51) but using twice the computational domain size so that $L_{c}=2 L_{0}=8 \pi$. We find that once again, at large times, the same travellingwaves with period $L=4 \pi$ emerge. Interestingly, in a further simulation using $L_{0}=L_{c}=8 \pi$, so that now the perturbation has the same wavelength as the wider domain, the waves are found to settle eventually to the same $4 \pi$-periodic travelling waves seen in the two previous simulations. From this set of three calculations, we conclude that the $k=0.5$ travelling-wave computed in figure 8 is stable and should be realisable in experiment. However, the travelling-wave at $k=0.25$ in figure 8 appears to be unstable and may not be observable. (We note that the wave profiles for $k=0.25$ closely resemble those for $k=0.1$ shown in figure $8 \mathrm{~b}$ ).

Despite the previous remarks, it may still be the case that the $k=0.25$ wave is stable, at least to small deviations. To investigate this, we use an initial condition which is a small perturbation of the travelling wave solution and take

$$
\begin{gathered}
f_{j}(\xi, 0)=\tilde{f}_{j}(\xi)+0.2 \cos \left(2 \pi \xi / L_{0}\right), \\
\Gamma_{j}(\xi, 0)=\tilde{g}_{j}(\xi)+0.2 \cos \left(2 \pi \xi / L_{0}\right)
\end{gathered}
$$

for $j=1,2$, where $\tilde{f}_{j}, \tilde{g}_{j}$ are the travelling wave solutions computed in section IV A. The result of a computation with $L_{0}=L_{c}=8 \pi$ is shown in figure 18 . Evidently the solution diverges from the $k=0.25$ ( $8 \pi$-periodic) solu-

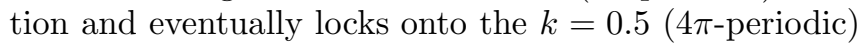
travelling-wave. This confirms that the $k=0.25$ solution is indeed unstable. We note in passing that a simulation with $L_{0}=L_{c}=4 \pi$ using initial condition (52) also locks onto the $4 \pi$-periodic travelling wave, providing further evidence of the stability of this solution. It appears,
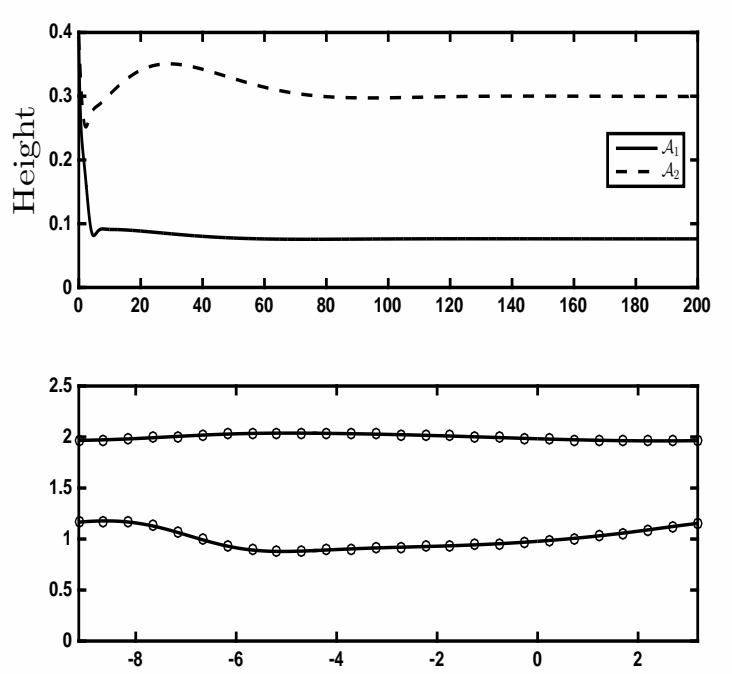

FIG. 17. Two-layer flow. Time-dependent simulation for the parameters in figure 8 and initial condition (51). $L_{0}=L_{c}=$ $4 \pi: \mathcal{A}_{1}, \mathcal{A}_{2}$ in the upper panel, and saturated profiles at $t=$ 200 in the lower panel. In the lower panel the travelling wave solution for $k=0.5$ computed using the method of section IV A is shown with circles.
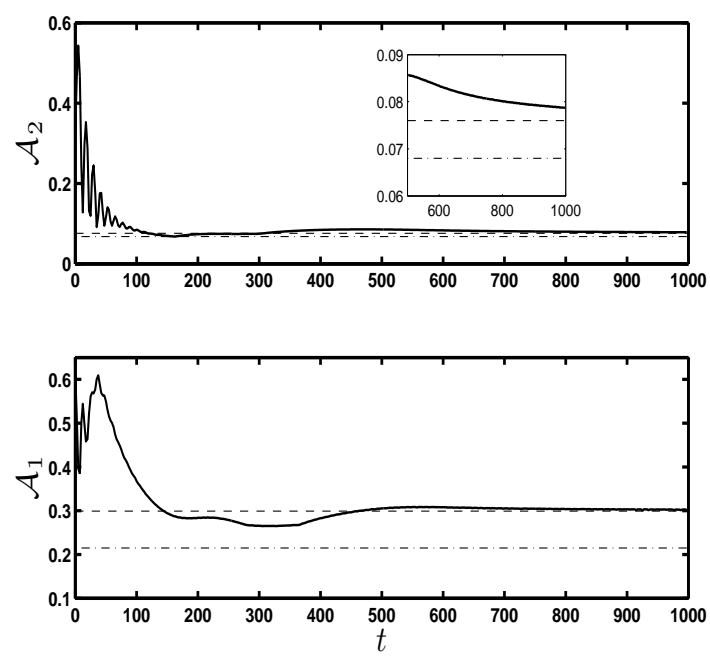

FIG. 18. Two-layer flow. Time evolution of the film wave heights for the parameters in figure 8 and initial condition (52). $L_{0}=L_{c}=8 \pi$. The $L=4 \pi$ and $L=8 \pi$ travelling wave heights are shown with broken and dot-dashed lines respectively. The inset shows a close-up at large time.

then, that at least part of the travelling-wave branch in figure 8 is stable, but that stability is lost as the period of the travelling-wave widens.

We now turn to a case where surfactant is present and examine the stability of the travelling waves for the case considered in figure 9. We consider first a travelling-wave at $k=0.7$. A simulation with $L_{c}=L_{0}=2 \pi / 0.7$ with initial condition (51) eventually settles to the travelling wave profile for $k=0.7$ predicted by the methods of 
section IV A. Repeating with initial condition (52), the solution returns to the same travelling-wave profile for $k=0.7$. Computing again using initial condition (52) but now taking two wave-periods over twice the domain size with $L_{c}=L_{0}=2 \pi / 0.35$, we find that the solution diverges from the $k=0.7$ travelling wave and the wave profiles develop to closely resemble the $k=0.35$ travellingwave solution with a clean region on the interface, as in upper panel of figure 10. The simulation fails to capture the profiles accurately because of the sharp variations in gradient in the profiles discussed in section IV A. To continue the simulations accurately, a prohibitively large number of Fourier modes would be required (we recall that the wave profiles in figure 10 required $N=1400$ modes), and consequently, an impractically small time step would be needed; moreover the inversion of the Jacobian in the Newton iterations at each time step becomes computationally very expensive. Simulations carried out with the surfactant diffusion present, taking the Péclet numbers to be finite in (44), produce qualitatively similar results, but suffer from similar difficulties when attempting to integrate to large times. Further evidence that the $k=0.7$ travelling-wave is unstable is provided in the next section. We conclude that although the travelling wave for $k=0.7$ is stable to same-wavelength perturbations, it is unstable to perturbations of twice the wavelength, and so it may not be observable in an experiment.

Finally, we mention the long-time behaviour for the initial value problem corresponding to the two-layer flow cases shown in figure 7 . Inside the island of unstable modes seen in figure $7(\mathrm{~b})$, we find that we may either latch onto a travelling-wave solution, once transients have decayed - this occurs when $\left(k, m_{2}\right)=(0.5,2.2)$ - or the time integration continues to a point where the surfactant concentration on the free surface reaches zero at a point, at which point the simulation is terminated - this occurs when $\left(k, m_{2}\right)=(1.0,0.2)$, for example. For the case when surfactant is on the interface and the free surface is clean, shown in figure 7(a), time-dependent simulations either break down when the surfactant reaches zero at a point - this occurs when $\left(k, m_{2}\right)=(0.5,0.5)$, for example - or continue to lock onto a travelling wave solution - this occurs when $\left(k, m_{2}\right)=(0.5,1.5)$ for example.

We now investigate the stability of travelling waves for three-layer flow. We focus initially on the clean case presented in figure 13. In a time-dependent simulation with $L_{0}=L_{c}=4 \pi$, and using initial condition (51), the surfaces profiles are found to saturate at large time to produce the $k=0.5$ travelling-wave profiles shown in figure $13(b)$. In contrast, for the surfactant-laden case in figure 14, quite different behaviour is found. The results of a simulation with initial condition (51) with $L_{0}=L_{c}=2 \pi$ is shown in figure 19. The time signals for the wave height on the two interfaces are similar to those shown for the free surface. Interestingly, rather than approach the travelling-wave profiles computed for $k=1.0$ in section IV A, the solution instead locks onto a periodic cycle corresponding to a periodic travelling wave, that is $(a)$
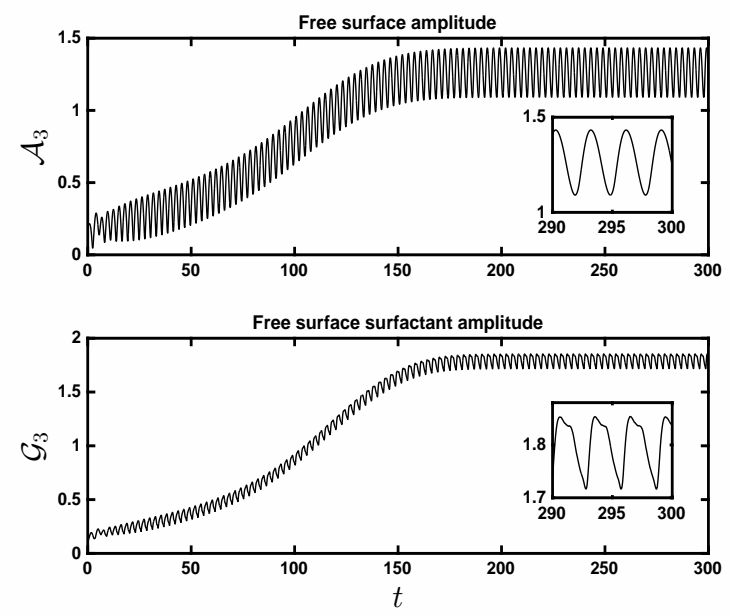

(b)
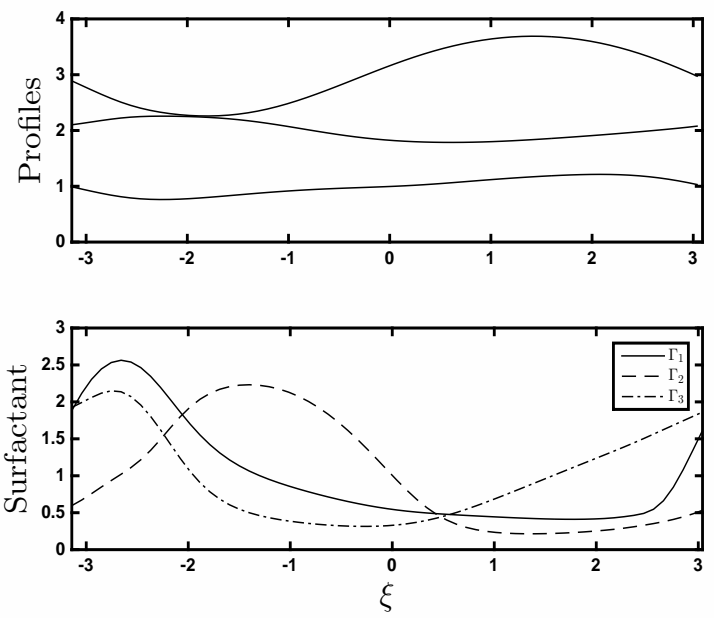

FIG. 19. Three-layer flow. (a) Time evolution of the free surface wave heights $\mathcal{A}_{3}=\max \left(f_{3}\right)-\min \left(f_{3}\right)$ and $\mathcal{G}_{3}=$ $\max \left(\Gamma_{3}\right)-\min \left(\Gamma_{3}\right)$ for the parameters in figure 14 and initial condition (51) with $L_{0}=L_{c}=2 \pi$. The insets show a close-up of the time signals. (b) The film and surfactant concentration profiles at $t=303.5$.

a travelling wave whose profile (within a reference frame translating at constant speed) is a periodic function of time. For illustration, the profiles at one time instant within the periodic cycle is shown in figure $19(b)$. It is striking that the free surface almost touches the second interface, and this remains the case throughout one time period. Periodic travelling waves have also been found in weakly-nonlinear models of surfactant-laden multilayer films in channel flow (e.g. [41]) and in core-annular flow (e.g. $[42,43])$.

The above observations suggest that it should be possible to visualise travelling-waves in both two-layer and three-layer systems in an experiment, but that other behaviours are also expected to be seen, including periodic 
travelling waves. Further comment on these findings are presented in the next section where we re-examine the stability problem by computing eigenvalue spectra.

\section{Stability of travelling waves}

The stability of the travelling wave solutions described above can be studied by applying Floquet theory (e.g. [44]). Working in the travelling wave frame, we perturb about a basic wave solution, writing,

$$
\left(f_{j}, g_{j}\right)=\left(\tilde{f}_{j}, \tilde{g}_{j}\right)+\epsilon \mathrm{e}^{\sigma \tau} \mathrm{e}^{\mathrm{i} p z} \sum_{n=-\infty}^{\infty}\left(a_{j n}, b_{j n}\right) \mathrm{e}^{\mathrm{i} n k z}
$$

for $j=1, \ldots, M$, where $M$ is the number of fluid layers, $k$ is the wavenumber of the travelling wave solution, $p$ is the real Floquet-Bloch parameter in the range $[0, k)$ and $\epsilon$ is a small parameter. The constant coefficients $a_{j n}$ and $b_{j n}$ are to be found. Spectral stability is assured if no point in the spectrum $\sigma(p)$ lies in the right half complex plane. Since the present problem is defined on an infinite domain, and the base waves $\tilde{f}_{j}(z)$ and $\tilde{g}_{j}(z)$ are periodic, the spectrum contains only a continuous part, that is the point spectrum of isolated eigenvalues is empty (see section 3.4.2 of Sandstede [45]). Substituting (53) into the travelling wave equations (33) and (44) and linearising, we obtain

$$
\begin{aligned}
\sigma \sum_{n=-\infty}^{\infty} a_{j n} \mathrm{e}^{\mathrm{i} n k z}=\sum_{l=1}^{M} \sum_{n=-\infty}^{\infty} & \left(D_{l j n}(z ; p) a_{l n}\right. \\
& \left.+E_{l j n}(z ; p) b_{l n}\right)
\end{aligned}
$$

and

$$
\begin{aligned}
\sigma \sum_{n=-\infty}^{\infty} b_{j n} \mathrm{e}^{\mathrm{i} n k z}=\sum_{l=1}^{M} \sum_{n=-\infty}^{\infty} & \left(F_{l j n}(z ; p) a_{l n}\right. \\
& \left.+G_{l j n}(z ; p) b_{l n}\right)
\end{aligned}
$$

both for $j=1, \ldots, M$, where $D_{l j n}(z ; p), E_{l j n}(z ; p)$, $F_{l j n}(z ; p), G_{l j n}(z ; p)$ are known periodic functions of $z$ which depend on the basic travelling wave solution and have period $L=2 \pi / k$. The formulae for these functions are lengthy and are suppressed in the interest of brevity. The stability problem is solved numerically for a chosen value of $p$ by collocating one period of the basic travelling wave with the $2 N+1$ equally-spaced points

$$
z_{j}=(j-1) h, \quad h=\frac{L}{N}, \quad j=1, \ldots, 2 N+1,
$$

and then truncating the infinite series in (54) and (55) at the finite level $n= \pm N$. Evaluating (54) and (55) at the collocation points yields a system of $8 N+4$ linear algebraic equations in the unknown coefficients $a_{1 n}, \ldots, a_{M n}$ and $b_{1 n}, \ldots, b_{M n}$, and $\sigma$. These may be gathered together to form a generalised eigenvalue problem for $\sigma$ of the generic form $\mathbf{A x}=\sigma \mathbf{B} \mathbf{x}$, where $\mathbf{A}$ and $\mathbf{B}$ are known matrices and $\mathbf{x}$ hosts the unknown coefficients. This eigenvalue problem is solved for each $p$ using the in-built Matlab routine 'eig'. To reduce the amount of computational effort required to determine the spectrum, we utilise the symmetry property

$$
D_{l j n}(z ; p)=D_{l j(-n)}^{*}(z ;-p),
$$

where the asterisk denotes the complex conjugate; $E_{l j n}$, $F_{l j n}$ and $G_{l j n}$ also have this symmetry. It follows that if

$$
\left\{\sigma, p, a_{1 n}, \cdots, a_{M n}, b_{1 n}, \cdots, b_{M n}\right\}
$$

is an eigenset then so is

$$
\left\{\sigma^{*},-p, a_{1(-n)}^{*}, \cdots, a_{M(-n)}^{*}, b_{1(-n)}^{*}, \cdots, b_{M(-n)}^{*}\right\} .
$$

Therefore, to complete the spectrum it is only necessary to consider values of the Floquet-Bloch parameter in the range $p \in[0, k / 2)$. We do this numerically by computing for a discrete set of $p$ points equally-spaced over this range. An approximation to the continuous spectrum follows as the union of all of the individual sets of $\sigma$ values which are computed at each of these individual $p$ values. We note that as a result of the translational invariance of the problem, the spectrum also includes the origin $\sigma=0$ (e.g. Sandstede [45]).

First we present results for the case of two layers, $M=2$. The numerical code for the Floquet stability calculation was checked in the case of uniform layer thicknesses (flat layer surfaces) against the linear stability calculation already discussed, and agreement between the two was confirmed. As a second check, we used our unsteady code from section IV C to integrate (33) and (44) forward in time starting from an initial condition given by (53) at $\tau=0$ with the $a_{j n}, b_{j n}$ corresponding to an eigenfunction for a chosen $p$. For $\tau>0$, we confirmed excellent agreement in the early stages of the calculation between the computed waveform and the linear approximation (53).

The stability spectrum for the travelling waves in figure 8 is shown in figure 20 for $k=0.5$ and $k=0.25$. In the former case all points in the spectrum lie in the left-half plane and the corresponding travelling-wave solutions are spectrally stable. This is consistent with the results of the unsteady simulations discussed in section IV C. For $k=0.25$ part of the spectrum lies in the right-half plane, implying linear instability. The points on the spectrum with largest real part $\sigma_{R}$ correspond to a non-zero value of $p$ (see the lower middle panel of figure 20) so that the most unstable mode is subharmonic, corresponding to a disturbance with wavelength longer than the wavelength of the base solution. Note also that $p=0$ is unstable so that the $k=0.25$ solution is unstable to perturbations of the same wavelength, in agreement with the findings of section IV C. Further computations reveal that if we restrict attention to superharmonic disturbances, $p=0$, this branch loses stability at $k=0.258$ via a Hopf bifurcation as a complex eigenvalue moves from the left to the 
(a)
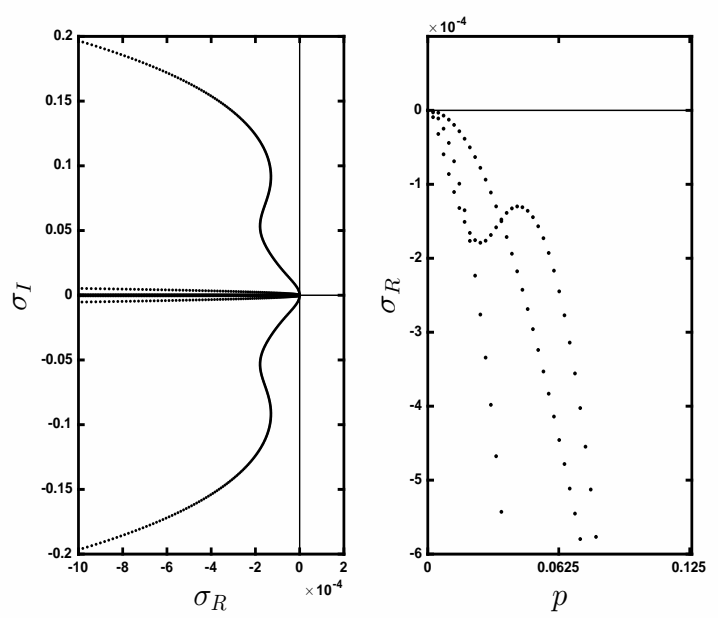

(b)
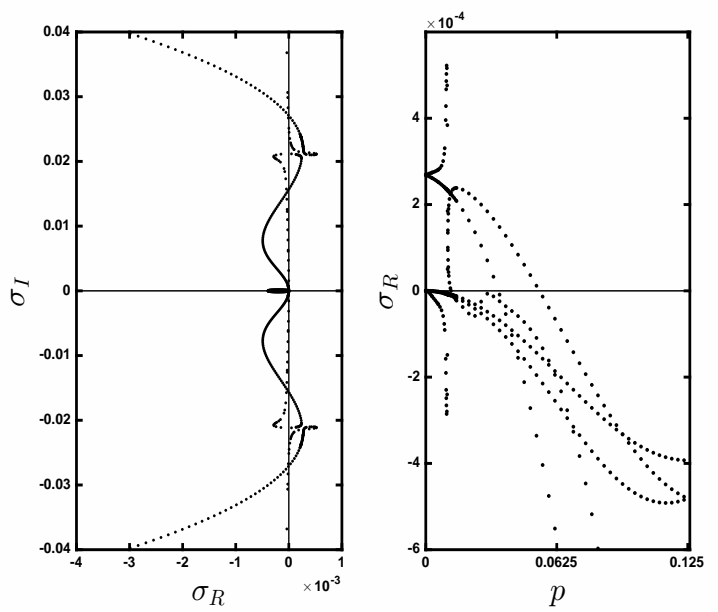

$(c)$
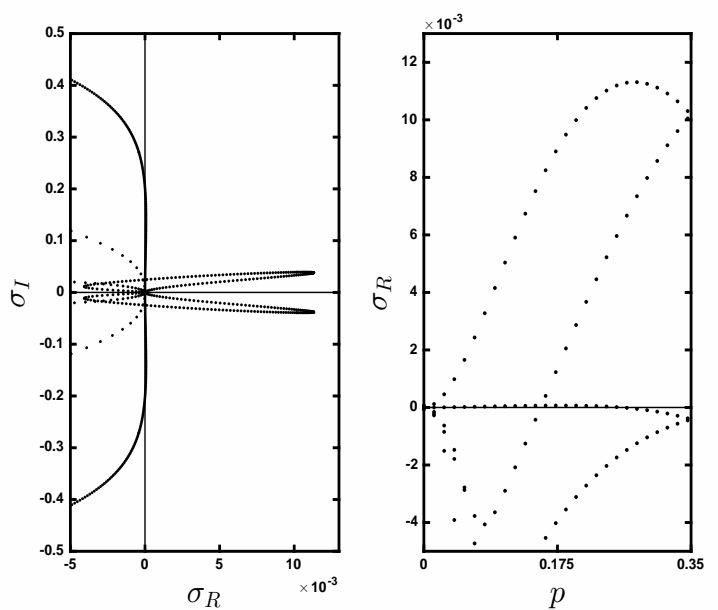

FIG. 20. Stability spectra for the two-layer flows studied in (a) figure 8 for $k=0.5$, and (b) figure 8 for $k=0.25$ and (c) figure 9 for $k=0.7$. right half complex plane. The existence of time-periodic travelling waves is then suspected; however, we have not been able to reach such a time-periodic solution via timedependent computations. These tend to lock instead onto the $k=0.5$ travelling wave solution (see section IV C).

The spectrum for the parameter case presented in figure 9 is shown in figure 20 for $k=0.7$. Since $p=0$ is stable, the wave solution is stable to superharmonic perturbations of the same wavelength, which agrees with the results of the time-dependent simulations in section IV C. However, the growth rate $\sigma_{R}$ at $p=0.35$ is evidently positive, so that the wave is unstable to subharmonic perturbations of twice the wavelength, which is again in agreement with the unsteady calculations reported above. We computed the stability spectra along the travelling wave branch down to $k=0.4$ and found that the waves are always unstable. This is in line with intuition since we expect the pulse solutions attained toward the end of this branch (figure $9 b$ ) to be unstable. This expectation is based on the observation that the flat parts of the films away from the localised pulse are linearly unstable. The three branches of travelling wave solutions identified in figure 11 was also investigated. Here branches 1 and 2 are everywhere unstable and branch 3 is everywhere stable.

Next we turn to the three layer problem, $M=3$, and investigate the stability of the travelling wave branches presented in figures 13 to 15 . For the surfactant-free case in figure 13, we find that the travelling wave solutions are unstable all the way along the branch. For the three wave branches shown in figure 14, branches 1 and 2 are everywhere unstable. On branch 3 the solutions are unstable all along the branch; however, if we restrict attention to superharmonic perturbations $(p=0)$, we find that the branch is stable up to $k=k_{H}$, with $k_{H}=1.19$, where stability is lost at a Hopf bifurcation when a complex eigenvalue crosses into the right half plane. When $k<k_{H}$ we therefore expect to find time-periodic solutions in a frame of reference moving with the steadily propagating wave. Such a periodic travelling-wave has already been discussed for this case (see figure 19). Further time-dependent calculations confirm that time-periodic travelling waves are found for $k<k_{H}$, while for $k>k_{H}$ the solution locks onto the steady travelling wave after a decaying oscillatory transient.

The conclusions of this section are that travelling-wave solutions of the type discussed in sections IV A and IV B for two-layer and three-layer flow may be spectrally stable, and hence should be observable in experiment.

\section{BOUNDARY-ELEMENT SIMULATIONS FOR STOKES FLOW}

We now relax the long-wave assumption and consider the time evolution of the two-layer or three-layer system under conditions of Stokes flow through numerical computation. The calculations are carried out using the 

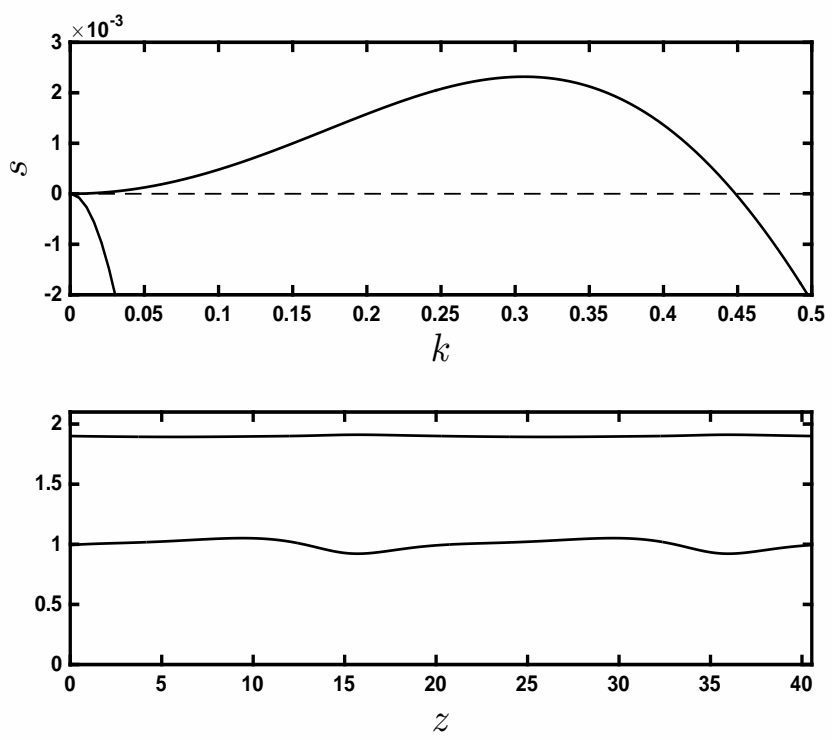

FIG. 21. Stokes flow simulation for clean two-layer flow with $\theta=\pi / 4, C a_{1}=C a_{2}=1, r_{2}=0.9, m_{2}=2.5, R_{2}=1, M a_{1}=$ $M_{2}=0.0$ using initial condition (58) with $L_{0}=L_{c}=2 \pi / 0.31$. Upper panel: Linear growth rates $s=\operatorname{Im}(k c)$ for Stokes flow. Lower panel: Two periods of the saturated wave travellingwave profiles for $k=0.31(L=20.27)$. The discretisation levels are $N_{S}=N_{I}=120$ and $\mathrm{d} t=7 \times 10^{-3}$.

boundary integral method for Stokes flow [e.g 46]. The formulation itself follows the comprehensive description provided by Pozrikidis [17] (see also [33] for a detailed description for multilayer film flows), and the reader is referred to these papers for particular details. The surfactant transport equation is integrated forward in time using the finite-volume method ([47]). In all of the computations to be described we discretize the interfaces and the free surface using straight elements. For two layer flow, we use $N_{I}, N_{S}$ elements on the interface and the free surface respectively. For three layer flow we use $N_{I_{1}}$, $N_{I_{2}}, N_{S}$ elements on interfaces 1,2 and the free surface respectively. With sufficient numbers of elements to ensure accuracy, the computations are expensive and consume very many hours of CPU time on a desktop machine. The number of boundary elements and the time step used in each calculation is quoted in the caption of the relevant figure.

Figure 21 shows the results of a boundary-element simulation for a clean two-layer system inclined at an angle $\theta=\pi / 4$. The computational domain is of length $L_{c}=2 \pi / k$ with $k=0.31$. The calculation was started at $t=0$ with the initial condition

$$
\begin{aligned}
y_{I} & =1+\epsilon a_{1} \cos \left(2 \pi x / L_{0}+\varphi_{1}\right), \\
y_{S} & =1+\epsilon a_{2} \cos \left(2 \pi x / L_{0}+\varphi_{2}\right), \\
\Gamma_{I} & =1+\epsilon b_{1} \cos \left(2 \pi x / L_{0}+\alpha_{1}\right), \\
\Gamma_{S} & =1+\epsilon b_{2} \cos \left(2 \pi x / L_{0}+\alpha_{2}\right),
\end{aligned}
$$

with $L_{0}$ taken equal to $L_{c}$, and with $\epsilon=0.2$. The subscripts $I$ and $S$ in (58) denote the interface and the free surface respectively. The film amplitudes and phase shifts are $a_{1}=0.21777, a_{2}=0.05963$, and $\varphi_{1}=3.12300$, $\varphi_{2}=0.36643$, The surfactant amplitudes and phase shifts are $b_{1}=0.90731, b_{2}=0.01132$ and $\alpha_{1}=0.10822$ and $\alpha_{2}=0.12752$. The values are chosen arbitrarily so that the initial condition represents a mixture of the available normal modes from the linear theory. The upper panel of figure 21(a) shows the stability graph found using the method described in section III. As the simulation progresses, the wave height on the free surface, $\mathcal{A}$, defined as the wave maximum minus the wave minimum, initially decays before starting to grow as the normal mode with the largest positive growth rate begins to dominate. Nonlinear effects come into play and eventually the wave height approaches a constant value and the waves saturate into travelling waves. Two periods of the travelling-wave profiles are shown in figure 21(b). It is noticeable that the interfacial deformation of the saturated wave is more marked than the free surface deformation. The waves propagate at speed $c_{w}=1.25$. The longwave theory of section IV A predicts the wave speed $c_{w}=1.27$. This is strikingly close, considering that this example does not lie within the longwave regime, which requires small capillary numbers (see section IV A); we note, however, that the longwave profiles do not closely resemble those shown in figure 21.

In figure 22 we show the results of a calculation for a two-layer flow with surfactant on the interface. The computational domain is $L_{c}=2 \pi / k$ with $k=0.65$. The initial condition is given by (58) at $t=0$ with $L_{0}=L_{c}$, and $a_{1}=0.11021, a_{2}=0.08050$, and $\varphi_{1}=0.21083$, $\varphi_{2}=0.29588$, and $b_{1}=0.60044, b_{2}=0.02622$, and $\alpha_{1}=-2.04395, \alpha_{2}=2.73905$. These values correspond to the normal mode with the largest growth rate, computed following the protocol described in section III. The upper panel of figure 22 (a) shows the stability graph found using the method described in section III. The maximum of the dominant growth rate is noticeably larger than those typically found for the smaller inclination angle $\theta=0.2$ used in section III. The lower panel shows the evolution of the wave height on the free surface, defined above, shown against dimensionless time $t$ (throughout this section the dimensionless scales are as given in section II). Included in this panel are two broken lines with gradients equal to that of the largest linear growth rate $s=0.04161$. Early in the simulation, this linear growth rate is captured accurately as the solid and the broken lines are almost coincident. Ultimately the waves saturate into travelling waves. The saturated profiles are shown in $22(b)$. The waves propagate at speed $c_{w}=1.27$. This compares favourably with the longwave prediction $c_{w}=1.22$; however, the travelling wave and surfactant profiles obtained from the longwave theory share the same qualitative features as those seen in figure $22(b)$ but do not agree quantitatively. In a separate boundary-element computation for the same parameter values, but with $k=1.5$, the wave height on 
(a)
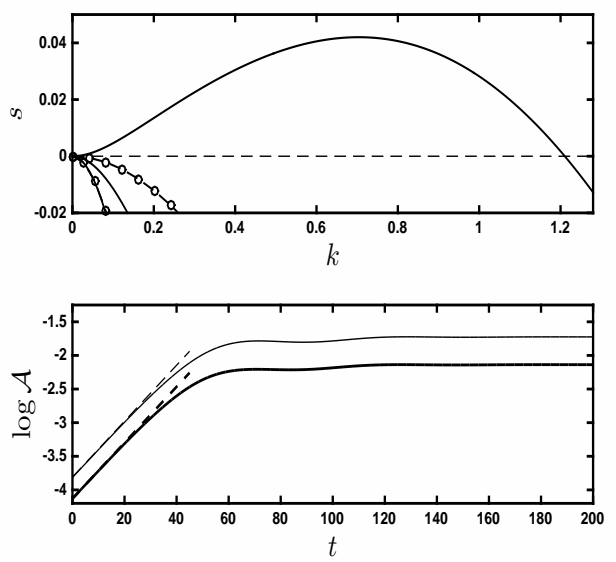

(b)
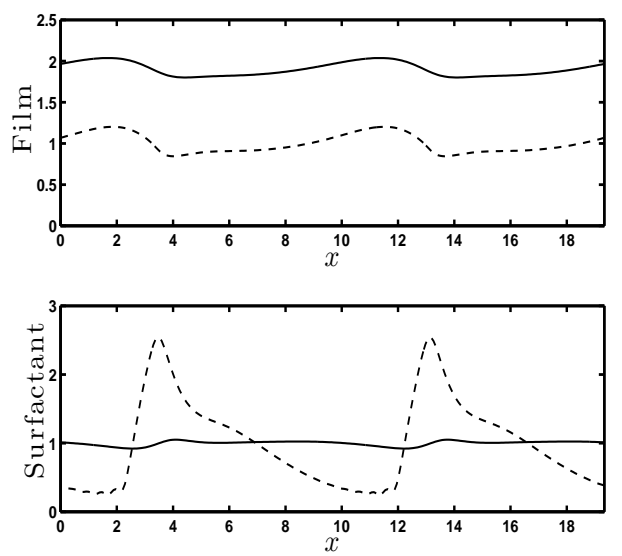

FIG. 22. Stokes flow simulation for two-layer flow with $\theta=\pi / 4, C a_{1}=C a_{2}=1, r_{2}=0.9, m_{2}=0.2, R_{2}=1$, $M a_{1}=0.5, M a_{2}=0.0$ using initial condition (58) with $L_{0}=L_{c}=2 \pi / 0.65$ : (a) (upper panel) Linear growth rates $s=\operatorname{Im}(k c)$ for Stokes flow and (lower panel) time evolution of $\log \mathcal{A}$, defined in the text, for $k=0.65$. The broken lines indicate the linear normal mode growth rates. The thick/thin lines are for the free surface/interface respectively. (b) Two periods of the saturated travelling-wave profiles for $k=0.65$ $(L=9.67)$. Interface/free surface profiles are shown with broken/solid lines. For computations, $N_{S}=N_{I}=120$ and $\mathrm{d} t=7 \times 10^{-4}$.

both the free-surface and the interface decays to zero, in accordance with the prediction of linear theory (figure $22(a)$, upper panel).

It might be expected that the nonlinear saturation identified in figure 22 will disappear if a larger computational domain with more fundamental periods is used. The CPU time required to complete such computations naturally escalates rapidly. We repeated the calculation for figure 22 using the same parameter values but on a domain of size $L_{c}=4 \pi / 0.65$ and with an initial condition given by (58) with $L_{0}=L_{c} / 2$ and with the right
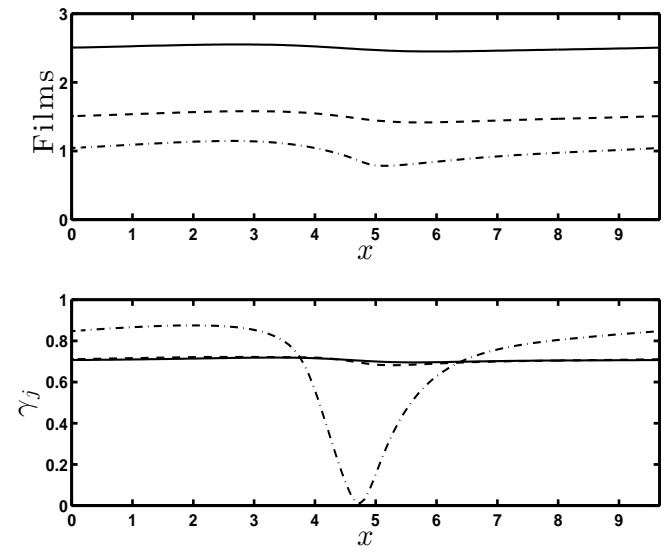

FIG. 23. Stokes flow simulation for three-layer flow with $\theta=$ $\pi / 4$ and $C a_{1}=C a_{2}=C a_{3}=1, r_{2}=0.5, r_{2}=1.5, m_{2}=$ $m_{3}=1, R_{2}=R_{3}=1, M a_{1}=M a_{2}=M a_{3}=1 / 2$ : The wave profiles and surface tension, $\gamma_{j}$, profiles profiles at $t=$ 40.0 for $k=0.65(L=12.57)$ for computation with $L_{0}=$ $L_{c}=L$. Interface $1 / 2$ and free surface profiles are shown with dot-dashed/dashed and solid lines respectively. For the computations, $N_{S}=N_{I}=120$ and $\mathrm{d} t=7 \times 10^{-3}$.

hand sides supplemented by an additional small perturbation of wavelength $L_{c}$. For this calculation, the nonlinear saturation previously identified does not occur but rather the surfactant on the interface reorganises itself so that the interfacial tension profile becomes zero at one point and the simulation fails. Qualititatively similar behaviour may also be observed for three-layer flow, as is illustrated in figure 23. The fact that the interfacial tension $\gamma_{1}$ touches zero indicates a breakdown in the physical model before this point in the simulation is reached. To investigate whether this breakdown has occurred as a result of the linear equation of state adopted for the surface tension in (4), we repeated the calculation in figure 23 , but using instead the nonlinear Langmuir equation of state (see, for example Pozrikidis [33], p. 234),

$$
\gamma_{j}=\gamma_{j c}\left(1+\Gamma_{\infty} \beta_{j} \log \left(1-\Gamma_{j} / \Gamma_{\infty}\right)\right),
$$

where $\beta_{j}=M a_{j} /\left(1+M a_{j}\right)=1 / 3$, and $\Gamma_{\infty}$ is the maximum surfactant packing concentration. We experimented with the three values $\Gamma_{\infty}=2,5,10$ and in all cases found that the same breakdown occurs and the surface tension reaches zero at a point on the lowermost interface. The effect of surfactant diffusivity was investigated by re-running the simulation with the linear equation of state (4) and taking $P e_{j}=10, j=1,2,3$ or $P e_{j}=100, j=1,2,3$. Once again the same qualitative breakdown occurs.

An example calculation for three layers is shown in figure 24. The initial condition is given by the obvious extension of (58) to three layers with $a_{1}=0.14629$, $a_{2}=0.07231, a_{3}=0.04694$, and $\varphi_{1}=-3.08458, \varphi_{2}=$ $2.84746, \varphi_{3}=2.88249$, and $b_{1}=0.70847, b_{2}=0.04396$, $b_{3}=0.02389$, and $\alpha_{1}=0.72337, \alpha_{2}=-0.09832$, 
(a)
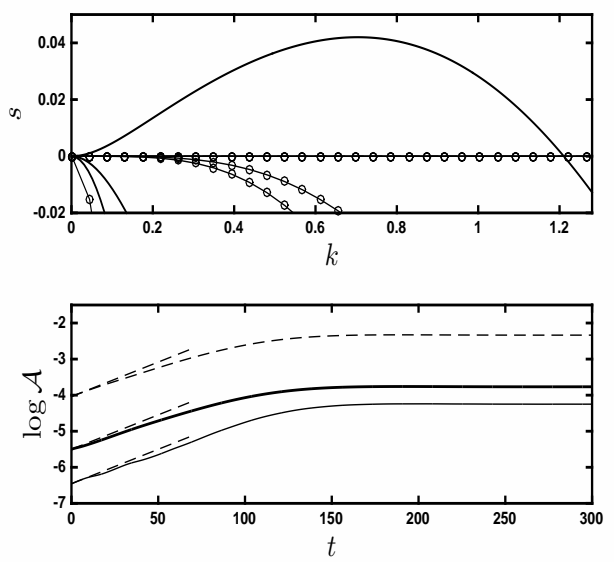

(b)
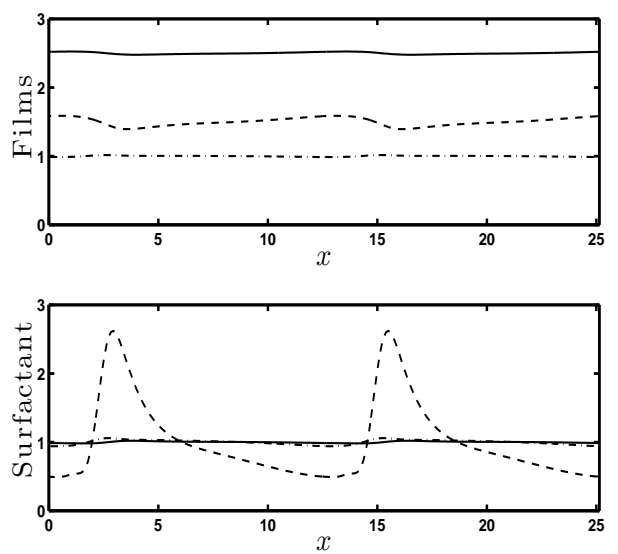

FIG. 24. Stokes flow simulation for three-layer flow with $\theta=\pi / 4$ and $C a_{1}=C a_{2}=C a_{3}=2, r_{2}=0.5, r_{2}=1.5$, $m_{2}=m_{3}=1, R_{2}=R_{3}=1, M a_{1}=0, M a_{2}=0.25$, $M a_{3}=0:(a)$ (upper panel) Linear growth rates $s=\operatorname{Im}(k c)$ for Stokes flow and (lower panel) time evolution of $\log A$, defined in the text, for $k=0.5$. The broken lines indicate the linear normal mode growth rates. The thick/thin lines are for the free surface/interface respectively. (b) Two periods of the saturated wave profiles for $k=0.5(L=12.57)$ for computation with $L_{0}=L_{c}=L$. Interface $1 / 2$ and free surface profiles are shown with dot-dashed/dashed and solid lines respectively. For the computations, $N_{S}=N_{I}=120$ and $\mathrm{d} t=7 \times 10^{-3}$.

$\alpha_{3}=-0.41960$. Here the subscripts $1,2,3$ refer to interface 1 , interface 2 and the free surface respectively. These values correspond to the dominant growth rate, indicated by the broken lines in the lower panel of figure 24(a). By about $t=200$, the solutions have settled into travelling waves propagating at speed $c_{w}=2.51$ (this compares with the longwave prediction at the same parameter values of $\left.c_{w}=2.5099\right)$. The surfactant concentration on interface 2 develops a much larger deviation from the base level than that on the other two surfaces, and reaches quite a large peak value $\left(\Gamma_{2}=2.6\right)$ which corresponds, according to (4), to the low interfacial tension $\gamma_{2}=0.2$.

\section{PHYSICAL CONTEXT}

We use recent experimental studies on multilayer flows ([48], [49]) as a guide to discuss likely physical values for our parameters. These experiments used predominantly water-glycerol mixtures with viscosities in the range $\mu=$ $15-260 \mathrm{mPa}$.s and density around $\rho=1200 \mathrm{~kg} \mathrm{~m}^{-3}$. The surface tension for each of these fluids (when in contact with air) was in the range $42-60 \times 10^{-3} \mathrm{Nm}^{-1}$ and in the absence of other measurements we will assume values in this range for our interfacial tensions. The angle of inclination of the plane was set at $\theta=\pi / 6$ radians, and the flow rates in the fluid layers was in the range $5-10 \times 10^{-6} \mathrm{~m}^{3} \mathrm{~s}^{-1}$.

Integrating the base flow velocity profiles in (8) we obtain the flow rate in the layer $i, Q_{i}=\int_{h_{i}}^{h_{i+1}} \overline{u_{i}}(y) \mathrm{d} y$. Note that a given choice of flow rates corresponds to a unique set of layer thicknesses ([39]). We select the typical experimental flow rates $Q_{1}=Q_{2}=Q_{3}=5 \times 10^{-6}$. Choosing mid-range values for the viscosity and surface tension, we find the layer thicknesses $0.52 \mathrm{~cm}, 0.78 \mathrm{~cm}$ and $1.02 \mathrm{~cm}$ for layers $1,2,3$ respectively so that $r_{2}=0.49$ and $r_{3}=0.44$, and obtain typical values for the Reynolds numbers and capillary numbers, $R e_{j} \equiv \rho_{j} h_{1} U_{s} / \mu_{j}=5.5 \times 10^{-5}$ and $C a_{j}=3.2$. The smallness of the Reynolds number lends credence to the assumption of Stokes flow in a theoretical analysis. The computed values of the layer thickness ratios and the capillary numbers are in line with the typical values chosen in section III and section $\mathrm{V}$.

If the film thicknesses are on the order of microns (such thicknesses have been realised in thin-film experiments, e.g. [50]), much smaller capillary numbers are possible. Taking the layer thicknesses to be on the order of $1 \mu \mathrm{m}$ in thickness, we obtain capillary numbers on the order of $10^{-3}$, corresponding to a long-wave parameter $\delta \approx$ 0.1 , which may be small enough to realise the long-wave regime discussed in section IV.

\section{DISCUSSION}

We have examined the flow of bilayer and trilayer flow of surfactant-laden viscous films down an inclined plane with the focus on studying flow stability and computing the nonlinear states which arise in the presence of instability. We have assumed zero Reynolds number throughout, so that inertia is absent, and studied the problem under conditions of Stokes flow and in the lubrication limit of long waves. In the latter case a simpler system of equations can be derived which is more amenable to analysis but which nonetheless preserves many of the important features and characteristics of the full Stokes system. We have derived the latter system for two or three layers, but 
the formulation easily generalises to any number of fluid layers.

In the basic state, the liquid layers have uniform thickness and the free surface and the interfaces between layers are all flat. Instability occurs in physical systems for which at least one of the upper layers is more viscous than a layer below it. Physical systems are also made unstable when surfactant is present on any of the interfaces between layers, or when it is present on the free surface and the uppermost layer is sufficiently thin in comparison to the other layer or layers. In the latter case, an otherwise stable clean configuration of layers may or may not remain stable when surfactant is added to the free surface, depending on the viscosity ratios of the fluids. A stable configuration of clean layers of equal thickness, whose viscosities decrease monotonically from the bottom layer upwards, is not destabilised by adding surfactant to the free surface. For specified values of the physical parameters, for a two-layer flow at most two or four possible normal modes can be unstable, and for a three-layer flow at most three of six possible normal modes can be unstable.

An unstable system supports travelling wave solutions which bifurcate from the neutral stability point, or points, where the growth rate of one or more of the normal modes vanishes. A clean two-layer flow has periodic travelling-wave solutions which evolve into pulses when the travelling-wave branch is followed to small wavenumber. Each surface exhibits a single pulse and both pulses travel in phase. A two-layer configuration with surfactant supports travelling-wave states with periodic bulges and capillary-ridge structures reminiscent of those seen, for example, in advancing contact lines (e.g. [51]) and in flow over topography (e.g. [37], [52]). In some cases, shocks develop in the surfactant profiles as a clean region appears over part of a wave period. Shocks are also observed in the spreading of a surfactant monolayer over a thin film (see for example [53, 54]) and in the gravity-driven motion of a surfactant-laden liquid drop over a precursor film ([55]). Pulse states are attained on travelling-wave branches at small wavenumber. For these pulses one of the interfaces is devoid of surfactant in the region behind the pulse. The flow of three clean liquid layers can also support pulse solutions. Three-layer flow with surfactant supports travelling-wave solutions in which two of the film surfaces are almost touching, and with the surfactant concentration on one of the surfaces possibly reaching a large peak value in the region of nearcontact. Pulse solutions are also possible with a single pulse on each surface each travelling at the same speed.

We examined the stability of the travelling-wave states by carrying out time-dependent simulations of the longwave system of equations from a specified initial condition. These demonstrated that travelling-waves for a clean system may be stable to perturbations with the same wavelength, or perturbations of larger wavelength. Waves in surfactant-laden systems were found in some cases to be stable to same wavelength disturbances but unstable to longer disturbances. In the case of a threelayer flow, these computations also revealed the existence of time-periodic travelling-waves for which the waves are time-periodic within a frame of reference travelling at a constant speed. Time-dependent simulations for Stokes flow carried out using the boundary-element method showed that, for both clean and surfactant-laden flows, travelling-wave states may be attained via nonlinear saturation after an initial transient, at least on sufficiently short computational domains. We presented evidence that this nonlinear saturation does not occur when the computational domain is widened to include more fundamental wave periods. Under conditions of Stokes flow, the breakdown of the physical model was observed for certain parameter values in which the agglomeration of surfactant forces the surface tension to zero at a single point. The breakdown is not removed either by including diffusivity in the surfactant transport model, or by adopting a nonlinear equation of state to describe the relationship between surfactant concentration and surface tension.

The stability results obtained via time-dependent simulations were reinforced by calculations of eigenvalue spectra for the nonlinear travelling-wave states carried out using Floquet analysis. These confirmed that the travelling-waves may be stable to perturbations of the same wavelength as themselves, but unstable to larger, subharmonic perturbations. In some cases, a travellingwave branch is spectrally stable sufficiently close to the bifurcation from the neutral stability point, but loses stability further along. In other cases travelling-wave branches are unstable all the way along.

In conclusion, we have found that physical systems of two or three liquid layers, which are unstable either due to viscosity stratification or to the presence of an insoluble surfactant, support travelling-wave states which should be observable in experiment. On this latter point, it is important to emphasise that where our analysis has identified that a certain travelling-wave state is unstable, this does not necessarily render it insignificant from a physical point of view. For example, the travelling pulse solutions are linearly unstable simply by virtue of the fact that the flat part of the film both upstream and downstream of the pulse is itself unstable. In fact we expect that these pulse solutions are actually convectively unstable and, as such, it should be possible to visualise them in the laboratory. It is well-known that travelling pulses are observed in experiments on single-film systems (see, for example, [56]). Determining the convective/absolute nature of the instability of our results lies beyond the scope of the present study, and will be considered in future work.

\section{ACKNOWLEDGEMENTS}

The work of MGB was supported by the EPSRC under grant EP/K041134/1. We thank the anonymous referees 
for their valuable comments which have helped to improve the manuscript.

\section{APPENDIX A: LINEAR STABILITY FOR STOKES FLOW}

To compute the growth rates for Stokes flow, we substitute the normal mode forms (16)-(17) into the Stokes equations and simplify to derive the zero Reynolds number Orr-Sommerfeld equation for the perturbation streamfunction,

$$
\phi_{j}^{(i v)}-2 k^{2} \phi_{j}^{\prime \prime}+k^{4} \phi_{j}=0 .
$$

No-slip at the wall demands that

$$
\phi_{1}=0, \quad \phi_{1}^{\prime}=0
$$

at $y=0$. Continuity of velocity across interface 1 requires

$$
A_{1} \bar{u}_{1}^{\prime}+\phi_{1}^{\prime}=A_{1} \bar{u}_{2}^{\prime}+\phi_{2}^{\prime}, \quad \phi_{1}=\phi_{2}
$$

at $y=1$, and across the interface 2 requires

$$
A_{2} \bar{u}_{3}^{\prime}+\phi_{3}^{\prime}=A_{2} \bar{u}_{2}^{\prime}+\phi_{2}^{\prime}, \quad \phi_{2}=\phi_{3}
$$

at $y=y_{20}=1+r_{2}$. The normal and tangential components of the stress balance (2) at interface 1 demand that

$$
\begin{gathered}
\left(3 k^{2} \phi_{1}^{\prime}-\phi_{1}^{\prime \prime \prime}\right)-m_{2}\left(3 k^{2} \phi_{2}^{\prime}-\phi_{2}^{\prime \prime \prime}\right)-\mathrm{i} k A_{1}\left(\bar{p}_{1}^{\prime}-\bar{p}_{2}^{\prime}\right) \\
+\frac{\mathrm{i} k^{3} A_{1}}{C a_{1}}=0
\end{gathered}
$$

and

$$
\begin{array}{r}
\left(\phi_{1}^{\prime \prime}+k^{2} \phi_{1}+A_{1} \bar{u}_{1}^{\prime \prime}\right)-m_{2}\left(\phi_{2}^{\prime \prime}+k^{2} \phi_{2}+A_{1} \bar{u}_{2}^{\prime \prime}\right) \\
-\mathrm{i} k \frac{M a_{1}}{C a_{1}} G_{1}=0
\end{array}
$$

respectively, with both evaluated at $y=1$. At interface 2 , the normal and tangential balances of stress are

$$
\begin{gathered}
m_{2}\left(3 k^{2} \phi_{2}^{\prime}-\phi_{2}^{\prime \prime \prime}\right)-m_{3}\left(3 k^{2} \phi_{3}^{\prime}-\phi_{3}^{\prime \prime \prime}\right)-\mathrm{i} k A_{2}\left(\bar{p}_{2}^{\prime}-\bar{p}_{3}^{\prime}\right) \\
+\frac{\mathrm{i} k^{3} A_{2}}{C a_{2}}=0
\end{gathered}
$$

and

$$
\begin{array}{r}
m_{2}\left(\phi_{2}^{\prime \prime}+k^{2} \phi_{2}+A_{2} \bar{u}_{2}^{\prime \prime}\right)-m_{3}\left(\phi_{3}^{\prime \prime}+k^{2} \phi_{3}+A_{2} \bar{u}_{3}^{\prime \prime}\right) \\
-\mathrm{i} k \frac{M a_{2}}{C a_{2}} G_{2}=0
\end{array}
$$

respectively, both at $y=y_{20}$. At the free surface, the normal and tangential components of the stress balance demand that

$$
m_{3}\left(3 k^{2} \phi_{3}^{\prime}-\phi_{3}^{\prime \prime \prime}\right)-\mathrm{i} k A_{3} \bar{p}_{3}^{\prime}+\frac{\mathrm{i} k^{3} A_{3}}{C a_{3}}=0,
$$

and

$$
m_{3}\left(\phi_{3}^{\prime \prime}+k^{2} \phi_{3}+A_{3} \bar{u}_{3}^{\prime \prime}\right)-\mathrm{i} k \frac{M a_{3}}{C a_{3}} G_{3}=0
$$

respectively, both evaluated at $y=y_{30}$.

The kinematic conditions (3) at the the three surfaces are

$$
A_{j}\left(\bar{u}_{j}-c\right)+\phi_{j}=0
$$

for $j=1,2,3$ at $y=1, y_{20}$ and $y_{30}$ respectively. Substituting (18) into the surface equation of state (4), we find $g_{j}=-M a_{j} G_{j}$ for $j=1,2,3$. The linearised forms of the surfactant transport equation (6) are

$$
G_{j}\left(\bar{u}_{j}-c\right)+\left(\bar{u}_{j}^{\prime} A_{j}+\phi_{j}^{\prime}\right)=\frac{\mathrm{i} k G_{j}}{P e_{j}},
$$

for $j=1,2,3$ at $y=1, y_{20}, y_{30}$ respectively. Henceforth we shall make the physically reasonable assumption that the surfactant diffusivities are negligible, so that the Péclet numbers are infinite and set $P e_{j}=\infty$ for all $j$.

The general solution to (60) is

$$
\begin{aligned}
\phi_{j}(y)=a_{j} \cosh (k y)+b_{j} \sinh (k y) \\
+c_{j} y \cosh (k y)+d_{j} y \sinh (k y),
\end{aligned}
$$

where the coefficients $a_{j}, b_{j}, c_{j}$ and $d_{j}$ are to be found. Substituting (72) into equations (61)-(71) we obtain a linear system of equations which we may compile to form the generalised eigenvalue problem for the complex wave speed $c$,

$$
\mathbf{A} \cdot \mathbf{w}=c \mathbf{B} \cdot \mathbf{w}
$$

where $\mathbf{w}$ contains the 18 unknowns $a_{i}, b_{i}, c_{i}, d_{i}, A_{i}, G_{i}$, $i=1,2,3$, and $\mathbf{A}$ and $\mathbf{B}$ are known $18 \times 18$ coefficient matrices with $\mathbf{B}$ a diagonal matrix with only six non-zero elements. Requiring $\operatorname{det}(\mathbf{A}-c \mathbf{B})=0$ to satisfy (73) we derive a sixth-order polynomial for $c$ implying in general the presence of six normal modes.
[1] P. W. Baumeister, Optical coating technology (SPIE Press, 2004).

[2] R. J. LaPorte, Hydrophilic polymer coatings for medical devices (CRC Press, 1997).

[3] T. B. Benjamin, "Wave formation in laminar flow down an inclined plane," J. Fluid Mech. 2, 554-574 (1957).

[4] C.-S. Yih, "Stability of liquid flow down an inclined plane," Phys. Fluids 6, 321-334 (1963).

[5] J. Liu, J. D. Paul, and J. P. Gollub, "Measurements of the primary instabilities of film flows," J. Fluid Mech. 
250, 69-101 (1993).

[6] S. Whitaker, "Effect of surface active agents on the stability of falling liquid films," Ind. Eng. Chem. Fund. 3, 132-142 (1964).

[7] S. Whitaker and L. O. Jones, "Stability of falling liquid films. effect of interface and interfacial mass transport," AIChE Journal 12, 421-431 (1966).

[8] S. P. Lin, "Stabilizing effects of surface-active agents on a film flow," AIChE Journal 16, 375-379 (1970).

[9] B. E. Anshus and A. Acrivos, "The effect of surface active agents on the stability characteristics of falling liquid films," Chem. Eng. Sci. 22, 389-393 (1967).

[10] W. Ji and F. Setterwall, "On the instabilities of vertical falling liquid films in the presence of surface-active solute," J. Fluid Mech. 278, 297-323 (1994).

[11] C. Pozrikidis, "Effect of surfactants on film flow down a periodic wall," J. Fluid Mech. 496, 105-127 (2003).

[12] M. G. Blyth and C. Pozrikidis, "Effect of surfactant on the stability of film flow down an inclined plane," J. Fluid Mech. 521, 241-250 (2004).

[13] T. W. Kao, "Role of viscosity stratification in the stability of two-layer flow down an incline," J. Fluid Mech. 33, 561-572 (1968).

[14] D. S. Loewenherz and C. J. Lawrence, "The effect of viscosity stratification on the stability of a free surface flow at low reynolds number," Phys. Fluids 1, 1686-1693 (1989).

[15] K-P. Chen, "Wave formation in the gravity-driven lowreynolds number flow of two liquid films down an inclined plane," Phys. Fluids 5, 3038-3048 (1993).

[16] W. Y. Jiang and S. P. Lin, "Enhancement or suppression of instability in a two-layered liquid film flow," Phys. Fluids 17, 054105 (2005).

[17] C. Pozrikidis, "Gravity-driven creeping flow of two adjacent layers through a channel and down a plane wall," J. Fluid Mech. 371, 345-376 (1998).

[18] W. Y. Jiang, B. Helenbrook, and S. P. Lin, "Inertialess instability of a two-layer liquid film flow," Phys. Fluids 16, 652-663 (2004).

[19] P. Gao and X-Y. Lu, "Mechanism of the long-wave inertialess instability of a two-layer film flow," J. Fluid Mech. 608, 379-391 (2008).

[20] M. Boutounet, P. Noble, and J.-P. Vila, "Roll-waves in bi-layer flows," Math. Models Meth. Appl. Sci. 22, 1250006 (2012).

[21] P. Gao and X-Y. Lu, "Effect of surfactants on the inertialess instability of a two-layer film flow," J. Fluid Mech. 591, 495-507 (2007).

[22] A. Samanta, "Effect of surfactants on the instability of a two-layer film flow down an inclined plane," Phys. Fluids 26, 094105 (2014).

[23] A. F. M. Akhtaruzzaman, C. K. Wang, and S. P. Lin, "Wave motion in multilayered liquid films," J. Appl. Mech. 45, 25-31 (1978).

[24] C. K. Wang, J. J. Seaborg, and S. P. Lin, "Instability of multi-layered liquid films," Phys. Fluids 21, 1669-1673 (1978).

[25] S. J. Weinstein and M. R. Kurz, "Long-wavelength instabilities in three-layer flow down an incline," Phys. Fluids 3, 2680-2687 (1991).

[26] I. Kliakhandler and G. Sivashinsky, "Kinetic alpha effect in viscosity stratified creeping flows," Phys. Fluids 7, 1866-1871 (1995).

[27] S. J. Weinstein and K. P. Chen, "Large growth rate in- stabilities in three-layer flow down an incline in the limit of zero reynolds number," Phys. Fluids 11, 3270-3282 (1999).

[28] W. Y. Jiang, B. T. Helenbrook, S. P. Lin, and S. J. Weinstein, "Low-reynolds-number instabilities in threelayer flow down an inclined wall," J. Fluid Mech. 539, 387-416 (2005).

[29] I. Kliakhandler and G. Sivashinsky, "Inertial effects and long waves in multilayer plane poiseuille flows," Phys. Scripta 1996, 90 (1996).

[30] I. L. Kliakhandler and G. I. Sivashinsky, "Viscous damping and instabilities in stratified liquid film flowing down a slightly inclined plane," Phys. Fluids 9, 23-30 (1997).

[31] E. S. Papaefthymiou, D. T. Papageorgiou, and G. A. Pavliotis, "Nonlinear interfacial dynamics in stratified multilayer channel flows," J. Fluid Mech. 734, 114-143 (2013).

[32] A. Adamson, Physical chemistry of surfaces (Wiley, New York., 1967).

[33] C Pozrikidis, "Instability of multi-layer channel and film flows," Adv. Appl. Mech. 40, 179-239 (2004).

[34] X. Li and C. Pozrikidis, "The effect of surfactants on drop deformation and on the rheology of dilute emulsions in stokes flow," J. Fluid Mech. 341, 165-194 (1997).

[35] D. Halpern and A. L Frenkel, "Destabilization of a creeping flow by interfacial surfactant: linear theory extended to all wavenumbers," J. Fluid Mech. 485, 191-220 (2003).

[36] D. J. Benney, "Long waves on liquid films," J. Math. Phys 45, 150-155 (1966).

[37] S. Kalliadasis, C. Bielarz, and G. M. Homsy, "Steady free-surface thin film flows over topography," Phys. Fluids 12, 1889-1898 (2000).

[38] A. M. Cazabat, F. Heslot, S. M. Troian, and P. Carles, "Fingering instability of thin spreading films driven by temperature gradients," Nature 346, 824-826 (1990).

[39] J. Thompson, Stability of a liquid thread and stability and nonlinear evolution of multilayer fluid flow (PhD Thesis. University of East Anglia., 2015).

[40] H.-H. Wei, "On the flow-induced marangoni instability due to the presence of surfactant," J. Fluid Mech. 544, 173-200 (2005).

[41] A. P. Bassom, M. G. Blyth, and D. T. Papageorgiou, "Nonlinear development of two-layer couette-poiseuille flow in the presence of surfactant," Phys. Fluids 22, 102102 (2010).

[42] A. P. Bassom, M. G. Blyth, and D. T. Papageorgiou, "Using surfactants to stabilize two-phase pipe flows of core-annular type," J. Fluid Mech. 704, 333-359 (2012).

[43] S. A. Kas-Danouche, D. T. Papageorgiou, and M. Siegel, "Nonlinear dynamics of core-annular film flows in the presence of surfactant," J. Fluid Mech. 626, 415-448 (2009).

[44] B. Deconinck and J. N. Kutz, "Computing spectra of linear operators using the floquet-fourier-hill method," J. Comp. Phys. 219, 296-321 (2006).

[45] B. Sandstede, "Stability of travelling waves," (2002), In: B. Fiedler, ed. Handbook of Dynamical Systems II, North-Holland.

[46] C. Pozrikidis, Boundary integral and singularity methods for linearized viscous flow (Cambridge University Press, Cambridge., 1992).

[47] S. Yon and C. Pozrikidis, "A finite-volume/boundaryelement method for flow past interfaces in the presence of surfactants, with application to shear flow past a viscous 
drop," Comput. Fluids 27, 879-902 (1998).

[48] D. Henry, J. Uddin, J. Thompson, M. G. Blyth, S. T. Thoroddsen, and J. O. Marston, "Multi-layer film flow down an inclined plane: experimental investigation," Exp. Fluids 55, 1-14 (2014).

[49] J. O. Marston, S. T. Thoroddsen, J. Thompson, M. G. Blyth, D. Henry, and J. Uddin, "Experimental investigation of hysteresis in the break-up of liquid curtains," Chem. Eng. Sci. 117, 248-263 (2014).

[50] M. M. J. Decré and J. C. Baret, "Gravity-driven flows of viscous liquids over two-dimensional topographies," J. Fluid Mech. 487, 147-166 (2003).

[51] SM Troian, E Herbolzheimer, SA Safran, and JF Joanny, "Fingering instabilities of driven spreading films," EPL
(Europhysics Letters) 10, 25 (1989).

[52] D. Tseluiko, M. G. Blyth, D. T. Papageorgiou, and J. M. Vanden-Broeck, "Electrified viscous thin film flow over topography," J. Fluid Mech. 597, 449 (2008).

[53] Michael S Borgas and James B Grotberg, "Monolayer flow on a thin film," J. Fluid Mech. 193, 151-170 (1988).

[54] O. E. Jensen and J. B. Grotberg, "Insoluble surfactant spreading on a thin viscous film: shock evolution and film rupture," J. Fluid Mech. 240, 259-288 (1992).

[55] J. V. Goddard and S. Naire, "The spreading and stability of a surfactant-laden drop on an inclined prewetted substrate," J. Fluid Mech. 772, 535-568 (2015).

[56] S. Kalliadasis, C. Ruyer-Quil, B. Scheid, and M. G. Velarde, Falling liquid films (Springer, London, 2011). 